\title{
Interplay of complete wetting, critical adsorption, and capillary condensation.
}

\author{
A. Drzewiński, ${ }^{1}$ A. Maciołek,${ }^{2,3,4}$ A. Barasiński, ${ }^{1}$ and S. Dietrich ${ }^{2,3}$ \\ ${ }^{1}$ Institute of Physics, University of Zielona Góra, \\ ul. Prof. Z. Szafrana 4a, 65-516 Zielona Góra, Poland \\ ${ }^{2}$ Max-Planck-Institut für Metallforschung, Heisenbergstr. 3, D-70569 Stuttgart, Germany \\ ${ }^{3}$ Institut für Theoretische und Angewandte Physik, Universität Stuttgart, \\ Pfaffenwaldring 57, D-70569 Stuttgart, Germany and \\ ${ }^{4}$ Institute of Physical Chemistry, Polish Academy of Sciences, \\ Department III, Kasprzaka 44/52, PL-01-224 Warsaw, Poland
}

\begin{abstract}
The excess adsorption $\Gamma$ in two-dimensional Ising strips $(\infty \times L)$ subject to identical boundary fields, at both one-dimensional surfaces decaying in the orthogonal direction $j$ as $-h_{1} j^{-p}$, is studied for various values of $p$ and along various thermodynamic paths below the critical point by means of the density-matrix renormalization-group method. The crossover behavior between the complete wetting and critical adsorption regimes, occurring in semi-infinite systems, are strongly influenced by confinement effects. Along isotherms $T=$ const the asymptotic power law dependences on the external bulk field, which characterize these two regimes, are undercut by capillary condensation. Along the pseudo first-order phase coexistence line of the strips, which varies with temperature, we find a broad crossover regime where both the thickness of the wetting film and $\Gamma$ increase as function of the reduced temperature $\tau$ but do not follow any power law. Above the wetting temperature the order parameter profiles are not slab-like but exhibit wide interfacial variations and pronounced tails. Inter alia, our explicit calculations demonstrate that, contrary to opposite claims by Kroll and Lipowsky [Phys. Rev. B 28, 5273 (1983)], for $p=2$ critical wetting transitions do exist and we determine the corresponding wetting phase diagram in the $\left(h_{1}, T\right)$ plane.
\end{abstract}

PACS numbers: 05.50.+q, 68.35.Rh, 68.08.Bc

\section{INTRODUCTION}

Far away from phase boundaries, i.e., deep in the onephase region, condensed matter is perturbed by confining walls only within a thin layer proportional to the bulk correlation length $\xi$, i.e., at most approximately $15 \AA$. This changes drastically if the thermodynamic state of the bulk system is moved towards the boundary of phase transitions between the bulk phases. If the phase transition is of second order the bulk correlation length diverges and so-called critical adsorption occurs, i.e., the perturbation due to the wall located at $y=0$ penetrates deeply into the bulk, resulting in an algebraic divergence of the thickness of the interfacial structure $\int_{0}^{\infty}\left(\rho(y)-\rho_{b}\right) d y /\left(\rho\left(0^{+}\right)-\rho_{b}\right) \equiv \Gamma /\left(\rho\left(0^{+}\right)-\rho_{b}\right) \rightarrow \infty$, where for a one-component fluid as a paradigmatic case $\rho_{b}$ is the bulk number density for a given temperature $T$ and chemical potential $\mu$, and $\rho(y)$ is the fluid number density profile 1, 2]. Moreover, the order-parameter profile $m(y) \equiv\left(\rho(y)-\rho_{c}\right) / \rho_{c}$, where $\rho_{c}$ is the bulk critical number density, is governed by a universal scaling function which decays exponentially on the length scale of $\xi$ in the direction $y$ normal to the surface [1, 2]. If the phase transition is of first order wetting phenomena occur as a result of a subtle interplay between the substrate potential, interactions among fluid particles, and entropic contributions, in particular interfacial fluctuations [3]. The thickness of the interfacial structure diverges too, but here with exponents which are determined by the decay exponents of the pair potentials between the fluid particles and of the substrate potential [3].
These two types of phenomena are of significant practical importance, in particular for the condensed phase being a fluidum near the gas-liquid transition, such as water near the thermodynamic coexistence with its vapor, or binary liquid mixtures such as water and hydrocarbons near liquid-liquid phase transitions. In this context various applications arise ranging from the use of colloidal suspensions [4] to petroleum recovery [5]. Wetting films are relevant in many types of liquid coating processes, such as lubrication and adhesion and also for microfluidics and nanoprinting [6, 7]. Critical adsorption plays an important role, e.g., for heterogeneous nucleation in supercritical solvents [8] or in micro- and nanofluidic systems in order to achieve wetting of these small structures [9].

A characteristic property of liquids is that first-order gas-liquid or liquid-liquid phase transitions end at critical points. Therefore critical adsorption and wetting must emerge from each other upon moving along the first-order phase boundary. This creates a puzzle. Liquids governed by dispersion forces (decaying asymptotically $\sim r^{-6}$ with distance $r$ ) belong to the Ising universality class [10], i.e., the critical exponents describing the singular behavior of various thermodynamic quantities and structural properties are those of the Ising universality class. This also holds at interfaces. Specifically, along bulk coexistence $\Delta \mu=\left(\mu-\mu_{0}(T)\right) /\left(k_{B} T_{c}\right)=0$ one has asymptotically [1, 2]

$$
\Gamma \sim|\tau|^{\beta-\nu}, \quad \Delta \mu=0,
$$

where $\tau \equiv\left(T-T_{c}\right) / T_{c}$ is the reduced deviation from the critical temperature $T_{c}$ and $\Delta \mu$ measures, in units 


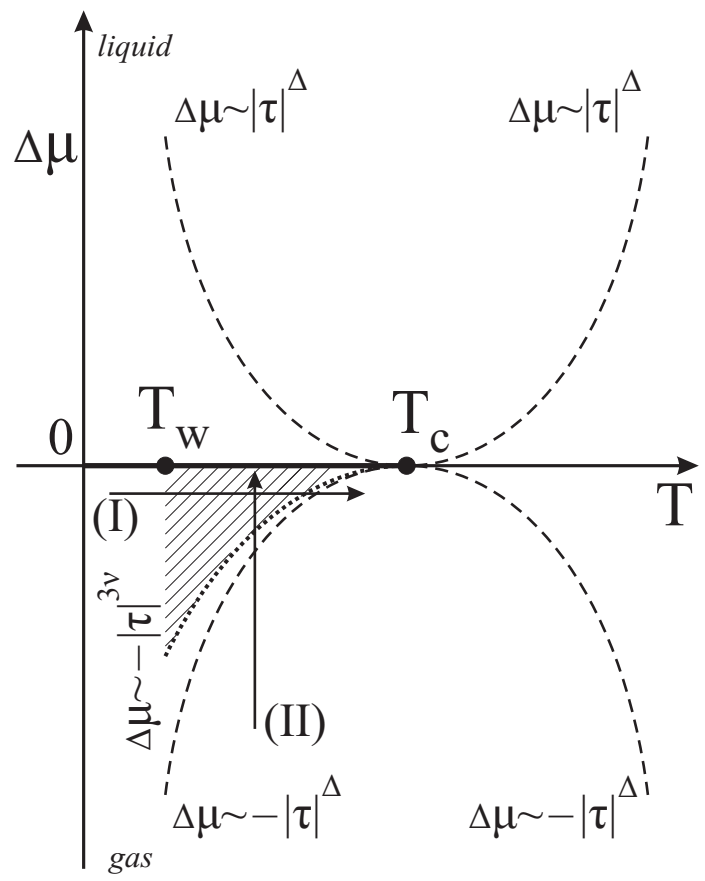

FIG. 1: Schematic drawing of the liquid-gas coexistence curve $\Delta \mu=0$ in the $(\Delta \mu, T)$ bulk phase diagram. The thick solid line indicates the bulk coexistence line. $T_{w}$ is the transition temperature for a critical wetting transition. Thermodynamic paths (I) and (II), along which the behavior of the adsorption is discussed in the main text, are displayed as well as various crossover lines. The hatched area is the complete wetting regime. Note that $3 \nu \simeq 1.89$ is larger than $\Delta \simeq 1.56$.

of $k_{B} T_{c}$, the deviation of the chemical potential from its value $\mu_{0}(T)$ at two-phase coexistence, whereas along the path $\tau=0$

$$
\Gamma \sim|\Delta \mu|^{(\beta-\nu) / \Delta}, \quad \tau=0,
$$

where the critical exponents $\nu, \beta$, and $\Delta$ take the values of the Ising model (in $d=3: \nu=0.6301(4), \beta=$ $0.32653(10)$, and $\Delta=3 \nu-\beta=1.564$ [1]). The latter universal singularity $\Gamma \sim|\Delta \mu|^{-0.194}$ is weaker than the non-universal one $\Gamma \sim|\Delta \mu|^{-1 / 3}$ for complete wetting [3], although according to the general renormalization group (RG) arguments the dispersion forces, which are responsible for this non-universal behavior, at first glance should give rise only to corrections to scaling (i.e., subdominant power laws). Naturally the question arises how the system manages to restore universality (i.e., dominance by the weaker universal power law) upon moving from the non-universal complete wetting behavior to the universal critical adsorption behavior. This issue has been addressed in Refs. [12, 13, 14] and the proposed scenario is such that upon approaching bulk coexistence, i.e., for $\tau$ fixed and $\Delta \mu \rightarrow 0^{-}$(see path (II) in Fig. 1), the system will always cross over ultimately to the complete wetting regime but such that upon approaching $T_{c}$ (i.e., for smaller $|\tau|$ ) this crossover occurs closer and closer to the coexistence curve. The proposed mechanism is based on the argument that long-ranged dispersion forces are relevant and can dominate if the thickness $\ell \sim|\Delta \mu|^{-1 / 3}$ of the wetting layer is much larger than the bulk correlation length $\xi \sim|\tau|^{-\nu}$. As described more closely in Sec. III for dispersion forces in $d=3$, this occurs if $|\Delta \mu| \ll|\tau|^{3 \nu}$ (see the hatched area in Fig. 11). Accordingly, upon approaching $T_{c}$ this complete wetting regime becomes vanishingly small. This expectation follows similar arguments as how dispersion forces become irrelevant for, e.g., two-point correlation functions in the bulk or for the critical adsorption profile itself, for which RG theory predicts exponential decays which, however, are dominated asymptotically by the algebraic decay due to the dispersion forces. Nonetheless the universal scaling functions, which capture the exponential decay, are expected to describe also the neighborhood of $T_{c}$ correctly, even in the presence of the irrelevant dispersion forces.

So far this puzzle has not been resolved satisfactorily by explicit calculations. The main reason is that mean field theory (MFT) as the natural theoretical starting point does not capture the correct $d=3$ exponents, which is essential for disentangling the different contributions and to describe the crossover. Within MFT, $\nu=\beta$ and the adsorption diverges only $\sim \ln |\Delta \mu|$. In systems governed by short-ranged forces only, within MFT both critical adsorption and complete wetting follow a divergence $\sim \ln |\Delta \mu|$ and therefore they cannot be distinguished; but the divergences differ beyond MFT. However, going beyond MFT is a difficult theoretical challenge, due to the spatial inhomogeneity.

There is also an urgent experimental need to resolve this issue. An ellipsometry study of gravity-thinned complete wetting layers in the binary-liquid mixture of cyclohexane and methanol [15] reports results which are not in accordance with the theoretical predictions. The data indicate the divergence of the film thickness upon approaching the bulk critical temperature characterized by a critical exponent which is distinct from the expected, critical exponent for the bulk correlation length. Scaling arguments have been put forward according to which the observed effective exponent is associated with a broad, intermediate scaling regime facilitating the crossover from complete wetting in the presence of dispersion forces to critical scaling [15]. Also recent neutron reflectometry data for the adsorption from alkane-perfluoroalkane mixtures at fluorophobic and fluorophilic surfaces [16] are not in agreement with the theoretical predictions. These authors have found that the behavior of the adsorption, as a function of temperature in the one-phase region upon approaching liquid-liquid coexistence with the composition well removed from the critical composition, can be represented by a power law with an exponent which differs from both that for complete wetting and that for critical adsorption [16].

The predictions described above for the crossover between the complete wetting regime of fluids with longranged interactions and the critical regime are based on general scaling arguments and on the analysis of simple 
models for the effective interface Hamiltonian which are supposed to describe the relevant physics at length scales much larger than the bulk correlation length [13]. Only few microscopic studies are available which test these ideas [12, 14]. Those are based on density functional methods and are of mean-field character, which in $d=3$ provides a correct description of complete wetting in the presence of dispersion forces but fails in the regime dominated by critical fluctuations. This makes it even more important to thoroughly analyze a model system which takes into account both the bulk and surface critical fluctuations of semi-infinite systems near $T_{c}$ as well as the interfacial fluctuations associated with wetting phenomena.

As a representative of the corresponding universality class, an Ising ferromagnet is well suited for such an analysis. In addition, addressing the issue in two spatial dimensions $(d=2)$ allows one to gain deep insight because the "exact" solvability provides, inter alia, the correct exponents. There is a recent experimental interest in such genuine two-dimensional systems in the context of proteins immersed in a fluid two-component lipid membrane which is near phase separation, including a critical point belonging to the $d=2$ Ising universality class [17, 18]. If two such proteins are close to each other the structural properties of the membrane between them can be described in terms of the strips studied here, giving even rise to interesting effective interactions between the proteins.

In the present analysis we use the density-matrix renormalization group (DMRG) method [19, 20, 21, 22] to numerically investigate the behavior of the adsorption in a two-dimensional $(d=2)$ Ising model with short-ranged as well as long-ranged surface fields. The DMRG method provides essentially exact numerical results for thermodynamic quantities and correlation functions, including the magnetization profiles. This allows us to study systematically the adsorption properties along various thermodynamic paths and thus to test the aforementioned predictions of the mesoscopic, effective interface Hamiltonian approaches. The DMRG method is based on the transfer matrix approach and it is a numerically very efficient iterative truncation algorithm for constructing the effective transfer matrices for large systems. The method was originally developed by White [19] for the diagonalization of quantum spin chains. It was then adapted by Nishino 20] to two-dimensional classical systems. The DMRG method allows one to study strips that are infinitely long with widths up to $L=700$ lattice constants with arbitrary surface and bulk fields. The comparison with exact results in the case of a vanishing bulk field and in the presence of contact surface fields shows that the DMRG method provides a very high accuracy for a broad range of temperatures.

Because the method requires finite values of $L$ the influence of the distant surface on the structural properties near the surface under study cannot be neglected, in particular, near the critical point - even for strips as wide as $L=700$. On one side, this for the DMRG method unavoidable confinement of the system complicates the aforementioned crossover behavior due to finite-size effects and capillary condensation. On the other side, the interplay between complete wetting, critical adsorption, and capillary condensation do not appear to have been discussed before, although it might be of relevance for adsorption-induced colloidal aggregation in binary liquid mixtures 23, 24]. Our approach is naturally suited for investigating interesting and relevant aspects of these important phenomena.

The paper is organized as follows. In Sec. II theoretical predictions for semi-infinite systems are summarized. In Sec. III we describe the microscopic model and the method. Numerical results for the phase diagram of the present model are described in Sec. IV] In Sec. V we determine the wetting temperature for different ranges of the boundary fields. Results for the magnetization profiles, the thickness of the wetting layer, and the adsorption along the isotherms and along the line of the pseudo-phase coexistence are reported in Sec. VI] Section VII summarizes and concludes our results.

\section{DESCRIPTION OF SEMI-INFINITE SYSTEMS}

A useful global characterization of the interfacial structure near a single wall is provided by the coverage $\Gamma$ defined as the excess number of fluid particles per area adsorbed on the confining substrate:

$$
\Gamma=\int_{0}^{\infty}\left(\rho(y)-\rho_{b}\right) d y
$$

where $\rho_{b}$ is the bulk number density for a given temperature $T$ and chemical potential $\mu$; here the fluid number density profile $\rho(\mathbf{r}) \equiv \rho(y)$ is assumed to vary only in the direction normal to the wall located at $y=0$.

\section{A. Complete wetting}

If the substrate potential is sufficiently strong there is a wetting transition temperature $T_{w}$ such that, if the bulk gas phase approaches gas-liquid coexistence $\mu_{0}(T)$ along isotherms at temperatures $T>T_{w}, \Gamma$ diverges due to complete wetting, i.e., a macroscopically thick wetting film is formed. The equilibrium thickness $\ell_{0}$ of the wetting film can be defined as

$$
\ell_{0}=\Gamma /\left(\rho_{l}-\rho_{g}\right),
$$

where $\rho_{l}$ and $\rho_{g}$ are the bulk number densities of the liquid and gas phase, respectively, at coexistence. In magnetic language the fluid is an Ising ferromagnet, the gas phase corresponds to the spin down phase, the liquid phase to the spin up phase, and the difference between the substrate potential and its analogue for fluidfluid interactions to a surface field; the undersaturation 
$\Delta \mu=\left(\mu-\mu_{0}(T)\right) /\left(k_{B} T_{c}\right)$ is proportional to the bulk field $H$.

In the complete wetting regime the increase of the adsorption upon approaching bulk coexistence can be described as [3]

$$
\Gamma(\Delta \mu \rightarrow 0, T) \sim|\Delta \mu|^{-\beta_{s}^{c o}}, \quad T_{w}<T<T_{c}
$$

The exponent $\beta_{s}^{c o}$ for this surface quantity depends on the form of the fluid-fluid and substrate-fluid forces as well as on the spatial dimension $d$. In $d=3, \beta_{s}^{c o}$ is non-universal; $\beta_{s}^{c o}=0$ (i.e., $\sim \ln \Delta \mu$ ) for short-ranged forces whilst $\beta_{s}^{c o}=1 / p$ for wall-fluid and fluid-fluid pair potentials decaying as $r^{-(d+p)}(p=3$ for nonretarded dispersion forces). Because the upper critical dimension for complete wetting with long-ranged forces is $d_{s}^{*}=3-4 /(p+1)<3$ [3, 25, 26] these mean-field exponents $\beta_{s}^{c o}=1 / p$ are not altered by interfacial fluctuations. For short-ranged forces, i.e., $p \rightarrow \infty$ one has $d_{s}^{*}=3$ so that fluctuations matter in $d=3$, but it turns that for complete wetting they change only the amplitude of the thickness of wetting film [3].

In $d=2$ interfacial fluctuations in the wetting films are much stronger and $2=d<d_{s}^{*}$ for both short-ranged and dispersion forces ( $p=4$ for the latter in $d=2$ ). Accordingly the complete wetting exponent takes a universal value which turns out to be given by $\beta_{s}^{c o}=1 / 3$, provided $p \geq 3$ so that fluctuations dominate [25, 26]. From the point of view of an effective interface Hamiltonian this latter universality of $\beta_{s}^{c o}$ is due to the entropic effects of the unbinding interface which give rise to an effective repulsive interaction for the gas-liquid interface, taken to be located on average at $y=\ell$, which decays $\sim \ell^{-\kappa}$ with $\kappa=2(d-1) /(3-d) ; \kappa(d=2)=2$. If this entropic repulsion dominates the effective interaction contribution $\sim \ell^{-(p-1)}$, i.e., if $p>\kappa+1$ one finds $\beta_{s}^{c o}=1 / 3$ in $d=2$; this defines the so-called weak fluctuation regime for complete wetting. According to this argument, for $p<3$, one has $\beta_{s}^{c o}=1 / p$. The considerations leading to the above predictions are valid only if the equilibrium wetting film thickness $\ell_{0}$ is much larger than the bulk correlation length $\xi$, i.e., $\ell_{0} \gg \xi$.

\section{B. Critical adsorption}

Near a critical point $T_{c}$, a confining wall generically provides an effective surface field $h_{1}$ acting on the order parameter (OP) $m$ describing the continuous phase transition and leading to the so-called critical adsorption [1, 2, 27]. The ensuing decay of the OP profile $m(y) \equiv\left(\rho(y)-\rho_{c}\right) / \rho_{c}$, where $\rho_{c}$ is the critical density, follows the power law

$$
m(y) \sim y^{-\beta / \nu}, \quad T=T_{c},
$$

where $\beta$ and $\nu$ are the known critical exponents of the bulk OP and of the correlation length $\xi$, respectively. Off the critical point a crossover to the exponential decay

$$
m(y) \sim \exp (-y / \xi)
$$

takes place at the distance $y \sim \xi$ from the surface. This is described by universal scaling functions $P_{ \pm}$such that $m_{ \pm}(y, \tau, \Delta \mu)=m_{b}^{(0)} P_{ \pm}\left(y / \xi_{ \pm}(\tau, \Delta \mu), \Delta \mu|\tau|^{-\Delta}\right)$, where $m_{b}^{(0)}=m\left(y \rightarrow \infty, T<T_{c}, \Delta \mu=0^{-}\right)=m_{0}|\tau|^{\beta}<0$, $\tau=\left(T-T_{c}\right) / T_{c}$, and $\Delta$ is the so-called gap exponent; \pm corresponds to $\tau \gtrless 0$. Accordingly the adsorption $\Gamma_{ \pm}$ diverges as

$$
\Gamma_{ \pm}=m_{0}|\tau|^{\beta} \xi_{ \pm}(\tau, \Delta \mu) K_{ \pm}\left(\Delta \mu|\tau|^{-\Delta}\right)
$$

where $K_{ \pm}(x)=\int_{0}^{\infty}\left[P_{ \pm}\left(\tilde{y}_{ \pm}, x\right)-P_{ \pm}(\infty, x)\right] d \tilde{y}_{ \pm}$[28]. Near the critical point the bulk correlation length acquires the scaling form

$$
\xi_{ \pm}(\tau, \Delta \mu)=|\tau|^{-\nu} \Xi_{ \pm}\left(\Delta \mu|\tau|^{-\Delta}\right),
$$

where $\Xi_{ \pm}$are scaling functions. Since $\Xi_{ \pm}(0)=$ const $=$ $\xi_{0}^{ \pm}$one has

$$
\xi_{ \pm}(\tau, 0)=\xi_{0}^{ \pm}|\tau|^{-\nu}
$$

whereas $\Xi_{ \pm}(x \rightarrow \pm \infty) \sim|x|^{-\nu / \Delta}$ and thus at $\tau=0$

$$
\xi(0, \Delta \mu)=\xi_{0}^{(\mu)}|\Delta \mu|^{-\nu / \Delta} .
$$

Together with $K_{ \pm}(x \rightarrow 0)=$ const and $K_{ \pm}(x \rightarrow \pm \infty) \sim$ $|x|^{\beta / \Delta}$, this implies that upon approaching $\left(T_{c}, \mu_{0}\left(T_{c}\right)\right)$ along the path $\Delta \mu=0$ the adsorption diverges according to Eq. (11) whereas it diverges according to Eq. (2) along the path $\tau=0$. We note that both Eq. (1) and Eq. (2) are consistent with the scaling behavior of $\Gamma \sim m \xi$ due to $m \sim|\tau|^{\beta}$ and $\xi \sim|\tau|^{-\nu}$ for $\Delta \mu=0$ and due to $m \sim|\Delta \mu|^{1 / \delta}, \delta=\Delta / \beta$, and $\xi \sim|\Delta \mu|^{-\nu / \Delta}$ for $\tau=0$. Within MFT $\beta=\nu=1 / 2$ which results in a logarithmic divergence along both paths.

Equation (2) is expected to hold [13] also below $T_{c}$ for $|\Delta \mu| \gg|\tau|^{\Delta}$ on both the wetting and non-wetting side of the coexistence curve, i.e., if the argument of the scaling functions $\Xi_{ \pm}$approaches infinity so that the behavior of $\xi_{ \pm}$is governed by $\Delta \mu$ (Eq. (11)). This regime is referred to as the critical adsorption regime. Similarly, above $T_{c}$ Eq. (1) is expected to be valid within the regime $|\Delta \mu| \ll|\tau|^{\Delta}$, i.e., where the behavior of $\xi$ is governed by $\tau$ (Eq. (10)). The same should hold close to bulk coexistence below $T_{c}$. However, it has been argued [12, 13, 15] that even near the critical point longranged forces are important, in the sense that for $\tau$ fixed and $\Delta \mu \rightarrow 0^{-}$the system will always cross over to the complete wetting regime (Eq. (5)) although upon approaching $T_{c}$ (i.e., for smaller $\tau$ ) this crossover will occur closer and closer to the coexistence curve. The expectation for this scenario to hold is based on the argument that long-ranged forces are relevant and thus dominant for $\ell_{0} \gg \xi$. Since $\ell_{0} \sim|\Delta \mu|^{-\beta_{s}^{c o}}$ and $\xi \sim|\tau|^{-\nu}$, this leads to the condition $|\Delta \mu| \ll|\tau|^{\nu / \beta_{s}^{c o}}$, i.e., $|\Delta \mu| \ll \tau^{3 \nu}$ 
for dispersion forces in $d=3$. Thus on the gas side region between the coexistence curve $\Delta \mu=0$ and the curve $\Delta \mu=$ const $\times|\tau|^{\nu / \beta_{s}^{c o}}$ the adsorption should be governed by wetting phenomena whereas the divergence of the adsorption $|\tau|^{\beta-\nu}$ according to Eq. (1) should be limited to the range $|\Delta \mu|^{1 / \Delta} \ll|\tau| \ll|\Delta \mu|^{\beta_{s}^{c o} / \nu}$. Furthermore, the effective interface Hamiltonian approach predicts that contrary to the critical adsorption regime, in the wetting dominated region the divergence should depend on the choice of the thermodynamic path taken. In particular, for any isotherm within this regime one expects $\Gamma \sim|\Delta \mu|^{-\beta_{s}^{c o}}$, but along a path $\Delta \mu=$ const $|\tau|^{x}$ with $x>\nu / \beta_{s}^{c o}$ one expects $\Gamma \sim|\tau|^{\beta-x \beta_{s}^{c o}}$. This follows from Eq. (4) with $\Delta \rho \sim|\tau|^{\beta}$ and $\ell_{0} \sim|\Delta \mu|^{-\beta_{s}^{c o}}$.

\section{Crossover phenomena}

These regimes described above are expected to give rise to rich crossover phenomena for the adsorption $\Gamma(\Delta \mu, T)$ upon crossing boundary lines between them along various thermodynamic paths. Two of them are particularly relevant for the present work: (i) isotherms $T=$ const $<T_{c}$ with $\Delta \mu \rightarrow 0^{-}$(see path (II) in Fig. 11) and (ii) a path parallel to the coexistence curve on the gas side with a small undersaturation $\Delta \mu=$ const $<0$ and with $T \rightarrow T_{c}$ (path (I) in Fig. 1).

Along an isotherm $T=$ const $>T_{w}$ and below the curve $\Delta \mu \sim-|\tau|^{\Delta}$ (see Fig. 1) $\Gamma$ increases as $|\Delta \mu|^{(\beta-\nu) / \Delta}$ upon approaching bulk coexistence from the gas side, i.e., for decreasing $|\Delta \mu|$, until one enters the crossover region between the lines $\Delta \mu=-$ const $|\tau|^{\Delta}$ and $\Delta \mu=-$ const $|\tau|^{\nu / \beta_{s}^{c o}}$ in which $\Gamma$ increases further; but therein no specific and well defined power law can be expected. Finally, when the path crosses the curve $\Delta \mu=-$ const $|\tau|^{\nu / \beta_{s}^{c o}}$ it enters the regime governed by wetting phenomena so that the adsorption should diverge as $|\Delta \mu|^{-\beta_{s}^{c o}}$. For $\mu=\mu_{0}$ the wall is wet and the adsorption is infinite (provided $T_{w}<T \leq T_{c}$ ).

The behavior of the adsorption along a path parallel to the coexistence curve is expected to be equally rich. Let us consider the case that the wetting transition at coexistence is continuous at $T=T_{w}$. According to the theory of wetting phenomena [3] the adsorption $\Gamma$ increases smoothly to some finite value (because $|\Delta \mu|>0$ ) upon approaching the wetting temperature $T_{w}$. This increase is governed by the scaling laws with respect to $\Delta \mu$ and $\tau$ which are associated with critical wetting. Upon a further increase of the temperature the adsorption should slightly decrease because $\Gamma=\ell_{0} \Delta \rho, \ell_{0}=$ const due to $\Delta \mu=$ const, and $\Delta \rho=\rho_{l}-\rho_{g} \sim|\tau|^{\beta}$ until the crossover line $\Delta \mu=-$ const $|\tau|^{\nu / \beta_{s}^{c o}}$ to the critical adsorption regime is reached. There $\Gamma$ should increase $\sim|\tau|^{\beta-\nu}$ until the next crossover line $\Delta \mu=-$ const $|\tau|^{\Delta}$ is encountered beyond which $\Gamma$ as a function of $\tau$ should saturate at a certain large value $\sim|\Delta \mu|^{(\beta-\nu) / \Delta}$. Finally, after passing the right branch of the crossover line $\Delta \mu \sim-\tau^{\Delta}$ (above $T_{c}$ ) the adsorption should decrease $\sim \tau^{\beta-\nu}$ upon increasing $\tau$.

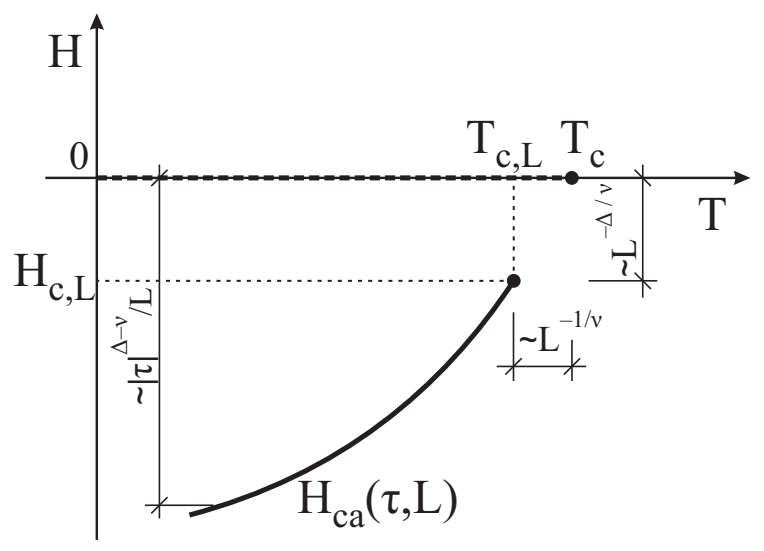

FIG. 2: Scaling behavior of the capillary condensation line $H_{c a}(\tau, L)$ near bulk criticality $\left(T=T_{c}, H=0\right)$. For $\tau=\left(T-T_{c}\right) / T_{c} \neq 0$ and $L \rightarrow \infty, H_{c a}(\tau, L)$ approaches bulk coexistence $H=0$ as $|\tau|^{\Delta-\nu} / L$ and the capillary critical point $\left(T_{c, L}, H_{c, L}\right)$ approaches $T_{c}$ and $H=0$ as $L^{-1 / \nu}$ and $L^{-\Delta / \nu}$, respectively. The latter is valid for strong surface field and takes the form $L^{-\left(\Delta-\Delta_{1}\right) / \nu}$, where $\Delta_{1}$ is the surface gap exponent, for weak $h_{1}$ [46]. Here $T_{c, L}<T_{c}$. Note the upward bent of the capillary condensation line; this feature is due to $\Delta-\nu<1$ (see, c.f., the text following Eq. (25)). However, this bent is weak: $\Delta-\nu=7 / 8=0.875$ in $d=2$ and $\Delta-\nu \simeq 0.936$ in $d=3$.

\section{MICROSCOPIC MODEL}

In this section we introduce the microscopic model within which we investigate quantitatively the crossover regimes described above. Specifically, we consider an Ising ferromagnet in a slit geometry subject to identical boundary fields. Contingent on the type of numerical approach we shall use, our results refer to $d=2$ strips defined on a square lattice of size $M \times L, M \rightarrow \infty$. The lattice consists of $L$ parallel rows at spacing $a$, so that the width of the strip is $L a$; in the following we set $a=1$. Successive rows are labeled by an index $j$. At each site, labeled $(k, j)$, there is an Ising spin variable taking the value $\sigma_{k, j}= \pm 1$. The boundary surfaces are located in the rows $j=1$ and $j=L$ and periodic boundary conditions (PBCs) are assumed in the lateral $x$ direction. Our model Hamiltonian for the strip with PBCs and $M \rightarrow \infty$ is given by

$$
\begin{aligned}
\mathcal{H}= & -J\left(\sum_{\left\langle k j, k^{\prime} j^{\prime}\right\rangle} \sigma_{j, k} \sigma_{j^{\prime}, k^{\prime}}\right. \\
& \left.+\sum_{j=1}^{L} V_{j, L}^{e x t} \sum_{k} \sigma_{k, j}+H \sum_{k, j} \sigma_{k, j}\right),
\end{aligned}
$$

where the first sum is over all nearest-neighbor pairs and the external potential is measured in units of $J>0$. 
$V_{j, L}^{e x t}=V_{j}^{s}+V_{L+1-j}^{s}$ is the total boundary field experienced by a spin in the row $j$; it is the sum of the two independent wall contributions. The single-boundary field $V_{j}^{s}$ is assumed to have the form

$$
V_{j}^{s}=\frac{h_{1}}{j^{p}}
$$

with $p>0$ and $h_{1}>0 . \quad H$ is a bulk magnetic field. According to Eq. (12) $h_{1}$ and $H$ are dimensionless.

This model can be viewed as being obtained from a $2 d$ lattice gas model mimicking a two-dimensional onecomponent fluid with a short-ranged interaction potential between the fluid particles and either short-ranged or long-ranged substrate potentials. The equivalence between the lattice gas and the Ising model implies the following relationships (see, e.g., Ref. [29]): the bulk magnetic field $H$ in the former is proportional to the deviation of the chemical potential from the bulk phase boundary $\mu_{0}(T)$ in the latter, i.e., $H \sim \Delta \mu$. The latticegas analogue of the number density in the fluid is related to the magnetization by $\rho=(m+1) / 2$, so that $\Delta \rho=\rho_{l}-\rho_{g} \sim 2 m_{b}$, where $m_{b}$ is the spontaneous magnetization. Finally $4 J$ corresponds to the strength of the attractive pair potential between the fluid particles, taken to be short-ranged so that in the lattice gas model it can be modelled by nearest neighbor interactions. $V^{e x t}$ is a combination of the substrate potential and the liquidliquid interaction. These relationships can be extended to binary liquid mixtures [30].

We recall that although there is no longer any true phase transition for finite $L$, in two-dimensional Ising strips with large $L$ there is still a line of extremely weakly rounded first-order transitions ending at a pseudocritical point the location of which in the plane $(H, T)$ spanned by the bulk field and the temperature $T$ depends on the character of the surface fields [31, 32]. For surfaces which prefer the same bulk phase, the phenomenon equivalent to capillary condensation takes place. The pseudo-phase coexistence between phases of spin up and spin down occurs along a line $H_{c a}(T, L)$, which is given approximately by the analogue of the Kelvin equation [33]:

$$
H_{c a}(T, L) \approx-\sigma(T) /\left(L\left|m_{b}^{(0)}(T)\right|\right),
$$

where $\sigma(T)$ is the interfacial tension (divided by $J$ ) between the coexisting bulk phases and $m_{b}^{(0)}(T)<0$ is the spontaneous bulk magnetization for $H=0^{-}$. For the $d=2$ Ising model, the surface tension is given exactly $\beta J \sigma(T)=2\left(K-K^{*}\right)$, where $K=J / k_{B} T$ and $K^{*}$ satisfies $\sinh 2 K \sinh 2 K^{*}=1$. The occurrence of thick wetting films of + spins at the two surfaces, for a thermodynamic bulk state corresponding to - spins, gives rise to nontrivial corrections to Eq. (14) which shift the condensation line to larger values of $|H|[32,[34]$. The pseudo-coexistence line ends at a pseudo-capillary critical point $\left(H_{c, L}, T_{c, L}\right)$ where $T_{c, L}\left(h_{1}, p\right)$ lies below the temperature $T_{c}$ of the bulk critical point. Its position as well as that of $H_{c, L}$ depends on $L$ and the strength and the range of the surface fields. For large $L$ and strong $h_{1}$ the shifts of the critical temperature and of the bulk field $H_{c, L}\left(h_{1}, p\right)$ are given by [32, 35] (see, c.f., Fig. 22)

$$
T_{c, L}-T_{c} \sim-L^{-1 / \nu}, \quad H_{c, L} \sim-L^{-\Delta / \nu}
$$

with $\nu=1$ and $\Delta=15 / 8$ for the two-dimensional Ising model.

\section{PHASE DIAGRAM}

In Fig. 3 we show the phase diagram for the present model calculated by using the DMRG method for a strip of width 340 with $h_{1}=0.8$ and three choices of the parameter $p$ describing the decay of the boundary field: $p=50,3$, and 2 . The case $p=50$ is expected to resemble the behavior corresponding to short-ranged surface forces. In this figure we display the various crossover lines discussed in Sects. [and $\amalg$ and the thermodynamic paths along which we have calculated the adsorption $\Gamma$. The pseudo-phase coexistence line, the crossover lines, and the adsorption have been determined for $2 d$ Ising strips within the DMRG method.

The thick solid line in Fig. 3 indicates the bulk phase coexistence line $\left(H=0, T<T_{c}\right)$ terminating at the bulk critical point $\left(H=0, T=T_{c}=[\ln (1+\sqrt{2})]^{-1} J / k_{B} \simeq\right.$ $2.269 \mathrm{~J} / k_{B}$ ). The symbols (open circles for $p=2$, open squares for $p=3$, and stars for $p=50$ ) show the pseudophase coexistence. It turns out that the pseudo-phase coexistence line for long-ranged boundary fields is located slightly further away from the bulk coexistence line, especially at lower temperatures, than the pseudo-phase coexistence line for the short-ranged boundary fields $(p=50)$. These pseudo-lines have been identified as those positions $(H, T)$ in the phase diagram where the total magnetization of the strip vanishes, i.e., $\sum_{j=1}^{L} m_{j}=0$ with $m_{j}=\left\langle\sigma_{k, j}\right\rangle$.

At fixed $T$ (fixed $H$ ) at those positions the free energy of the strip exhibits a maximum as a function of $H$ $(T)$ which is the rounded remnant of the nonanalyticity (kink) of the free energy at the first-order phase transition in the bulk $(L=\infty)$ system. As can be seen in Fig. 3, for $T<T_{c}\left(T>T_{c}\right)$ the line defined by the zeros of the total magnetization moves to less (more) negative values of $H$ upon increasing $T$. Determining the pseudocritical temperature $T_{c, L}$, where the pseudo-phase coexistence line ends, is difficult because in this quasione-dimensional system the critical point is not a sharp concept and one has to examine various criteria in order to estimate $T_{c, L}$. However, these different criteria, such as the maximum of the specific heat, or the erosion of the jumps in the adsorption and in the solvation force upon crossing the line $H_{c a}(T, L ; p)$, provide somewhat different estimates for $T_{c, L}$. Determining $T_{c, L}$ for different values of $p$ and $L$ is beyond the scope of the present analysis. 


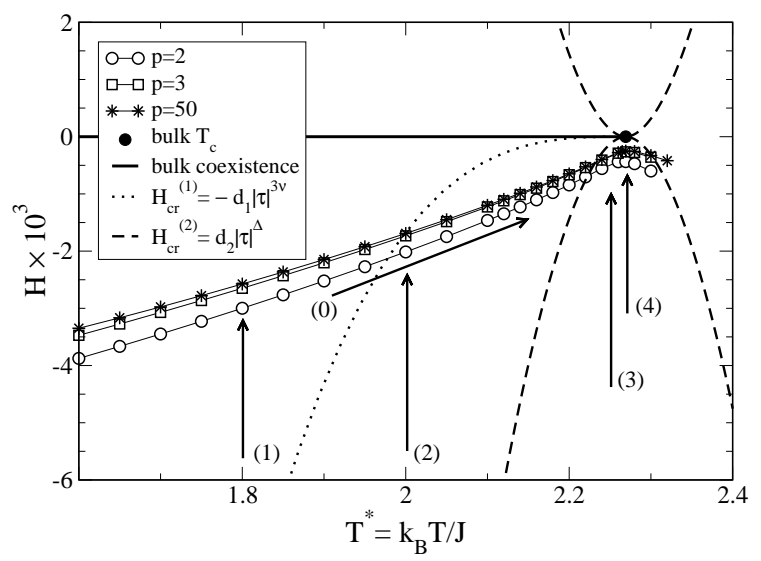

FIG. 3: Phase diagram for a $d=2$ Ising strip subject to identical boundary fields $V_{j}^{\text {ext }}$ (see Eq. (13)) obtained by using DMRG for a strip width $L=340$ and the amplitude $h_{1}=0.8$ for the boundary fields. The thick solid line indicates the bulk coexistence line. The lines interpolating the symbols represent the pseudo-phase coexistence lines $H_{c a}(T, L ; p)$ for three different values of the exponent $p$ governing the algebraic decay of the boundary fields: open circles correspond to $p=2$, open squares correspond to $p=3$, and stars correspond to $p=50$. Various crossover lines $H_{c r}^{(1)}$ and $H_{c r}^{(2)}$ with $d_{1}=1=d_{2}=1$ and $\nu=1$ and $\Delta=15 / 8$ in $d=2$, as discussed in the main text, are shown as well as the thermodynamic paths (0) - (4) along which the adsorption has been calculated. (1) - (4) are various isotherms and (0) runs parallel to the pseudo-coexistence line. Thus path (0) resembles (in a certain sense, see later) a path of type (I) in Fig. 1] whereas the paths $(1)-(4)$ do correspond to a path of type (II) in Fig. 1]

For the width $L=340$ and $h_{1}=0.8$ we approximately obtain $T_{c, L} \approx 2.2$; for more details concerning the shortranged case see Ref. [36].

We have investigated how the location of the pseudophase coexistence line $H_{c a}(T, L ; p)$ of capillary condensation changes as a function of $p$ for fixed width $L$, fixed amplitude $h_{1}$ of the surface field, and fixed temperature $T$. In Fig. 4 we plot the logarithm of the difference $H_{c a}(T, L ; p)-H_{c a}(T, L ; \infty)$ as a function of $p$ calculated for $h_{1}=0.8$ and (i) for several values of the temperature at fixed $L=300$ (Fig. 4(a)) as well as (ii) for several values of $L$ at fixed $T=1.8 \mathrm{~J} / k_{B}$ (Fig. 困(b)). (Note that $p=\infty$ corresponds to a pure surface contact field at $j=1, L$ (see Eq. (13)).) From these plots we can clearly distinguish two different regimes for the behavior of the pseudo-phase coexistence line, which are separated by a crossover region occurring for $1 \lesssim p \lesssim 3$. In both of these two regimes the shift $H_{c a}(T, L ; p)-H_{c a}(T, L ; \infty)$ varies exponentially but with different decay constants. Moreover, for $p \lesssim 1$ the shift relative to the short-ranged pseudo-phase coexistence line does not depend on the temperature; the dependence on the strip width $L$ becomes, for the range of $L$ considered here, very weak and finally negligible for $p \lesssim$ 0.25 , i.e., $H_{c a}(T, L ; p)-H_{c a}(T, L ; \infty) \approx B_{0} \exp (-A p)$ with $A \simeq 5.2$ and $B_{0} \simeq 1.5$. For $p \gtrsim 3$ one has $H_{c a}(T, L ; p)-H_{c a}(T, L ; \infty) \simeq B(L, T) \underset{\exp }{2}(-C p)$ with $C \simeq 0.723$. We note that, as expected, for $p$ fixed wider strips and higher temperatures give rise to smaller shifts of the pseudo-phase coexistence line.
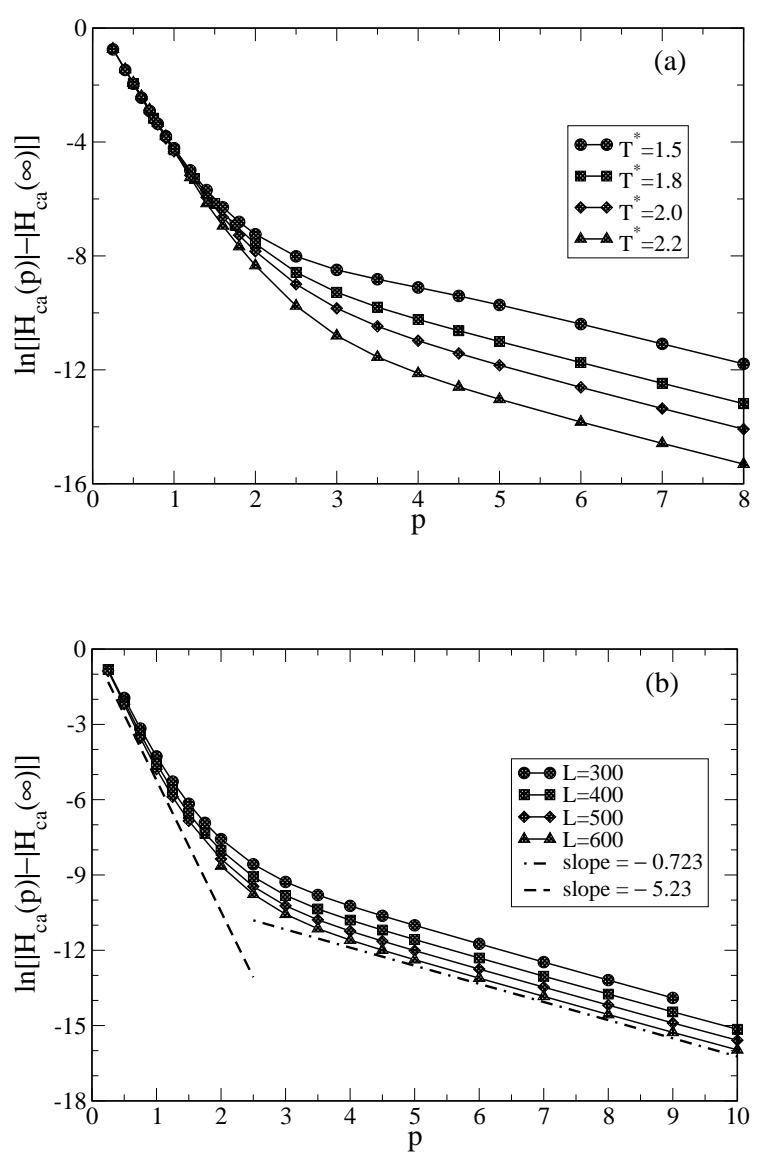

FIG. 4: The relative shift of the pseudo-coexistence line for Ising strips as function of the range of the boundary fields characterized by the decay exponent $p$ (Eq. (13)). The calculations are carried out by using the DMRG method for $h_{1}=0.8$ and (a) at fixed $L=300$ and for four temperatures $T^{*}=k_{B} T / J ;(\mathrm{b})$ at fixed temperature $\left(k_{B} T / J=T^{*}=1.8\right)$ and for four thicknesses $L$ of the strip.

\section{DETERMINATION OF THE WETTING TEMPERATURE FOR DIFFERENT RANGES OF THE BOUNDARY FIELDS}

It is important to assure that the range of temperatures, for which we perform our calculations of the adsorption, lies above the wetting temperature $T_{w}\left(p, h_{1}\right)$. For $h_{1}=0.8$ and a short range of the surface fields $(p=$ $\infty)$ the wetting temperature in the semi-infinite $d=2$ Ising system equals $T_{w}\left(p=\infty, h_{1}=0.8\right) \simeq 1.41 \mathrm{~J} / k_{B}$ and the wetting transition is second order. Adding the 
long-ranged tail to the boundary fields shifts the wetting temperature. Wetting transitions in $d=2$ in the presence of long-ranged substrate potentials have been discussed by Kroll and Lipowsky [37] within the continuum one-dimensional solid-on-solid model, with the conclusion that there is no wetting transition for fields which decay more slowly than $1 / j^{3}$. In this case their prediction is that the interface remains pinned to the wall at all finite temperatures. This conclusion has been supported by providing upper and lower bounds for the groundstate energy $E_{0}$ of the corresponding Schrödinger equation. Within this approach the existence of the boundstate solution of the Schrödinger equation $\left(E_{0}<0\right)$ corresponds to a localized interface. When the bound state ceases to exists, $E_{0} \rightarrow 0$ signals the critical wetting transition. The construction of upper and lower bounds (based on using an exponentially decaying trial wave function) shows that the ground-state energy has a non-zero value (i.e., there is no transition) for a finite potential strength. The case $p=2$ has been studied by Privman and Svrakić in Ref. 38] also within the SOS model; they argued that for attractive effective potentials decaying like $1 / r$ the wetting transition is no longer sharp (becomes rounded). For the $d=2$ Ising ferromagnet the wetting phenomena for the case of the marginal range $p=3$ have been studied within the DMRG approach [39] and by MC simulations [40, 41]. These studies have shown that the presence of long-ranged tails in the boundary fields decreases the wetting temperature with respect to the case of the short-ranged surface fields and that $T_{w}\left(p=3, h_{1}=0.8\right) \simeq 0.75 J / k_{B}$. Apart from Refs. [37, 38], the case $p<3$ has not been studied so far.

In order to check the predictions by Kroll and
Lipowsky, we investigate whether in the $d=2$ Ising ferromagnet with $p=2$ a wetting transition exists. In the finite systems we are studying, below the bulk critical temperature the wetting transition as a function of $h_{1}$ for any range $p$ of the boundary fields can be inferred from the so-called (weakly rounded in $d=2$ ) interface localization-delocalization (ILD) transition [32, 42] which occurs in strips with antisymmetric boundary fields, i.e., for $V_{j}^{e x t}=V_{j}^{s}-V_{L+1-j}^{s}$. This transition is the precursor of a wetting phase transition that occurs in the limit of infinite film thickness $(L \rightarrow \infty)$ at the critical curve $T_{w}\left(p, h_{1}\right)$. For $T<T_{w}\left(p, h_{1}\right)\left(T>T_{w}\left(p, h_{1}\right)\right)$ such an interface is bound to (unbound from) the walls [32, 42].

First, we note that $T_{w}\left(h_{1} \rightarrow 0, p\right) \rightarrow T_{c}$ for any $p$ (see Eq. (13)). On the other hand $h_{1 w}(T=0, p)$, which is the solution of the implicit equation $T_{w}\left(h_{1}, p\right)=0$ and denotes the critical surface field strength beyond which the system is wet even at $T=0$, shifts towards lower values upon decreasing $p$. There is no reason to expect a non-monotonic behavior of $T_{w}\left(h_{1}, p\right)$. Therefore, the gross features of the shape of the wetting transition line $T_{w}\left(h_{1}, p\right)$ for an arbitrary $p>1$ can be inferred from localizing the position of the wetting transition $h_{1 w}(T=$ $0, p)$ in the ground state. The ground-state energy of the system (in units of $J$ ) can be found directly from the Hamiltonian with a vanishing bulk field. Because the system is translationally invariant along a strip, it is sufficient to consider only the configurations of a single column.

In a partial wetting regime with antisymmetric surface fields there are only two coexisting states (all spins up or all spins down) with the energy (per the number $M$ of columns)

$$
\begin{aligned}
E_{p w}^{ \pm} & =-J(L-1) \mp h_{1} J\left(1-\frac{1}{L^{p}}+\frac{1}{2^{p}}-\frac{1}{(L-1)^{p}}+\ldots+\frac{1}{(L-1)^{p}}-\frac{1}{2^{p}}+\frac{1}{L^{p}}-1\right) \\
& =-J(L-1) .
\end{aligned}
$$

In a wet regime at least one interface between spin up and spin down configurations has to be present. For even $L$ the lowest-energy configuration is that for the state with the interface located in the middle of the strip. For $p \rightarrow$ $\infty$ (short-ranged case) $L-1$ degenerate states emerge with a single interface positioned at any of the rows but the ones closest to the surfaces. In general, the energy of a single column with an interface in the middle of a strip (spin up for $j=1, \ldots, L / 2$ and spin down for $j=L / 2+1, \ldots, L)$ is

$$
\begin{aligned}
E_{w e t} & =-J(L-3)-h_{1} J\left(1-\frac{1}{L^{p}}+\frac{1}{2^{p}}-\frac{1}{(L-1)^{p}}+\ldots+\frac{1}{(L / 2)^{p}}-\frac{1}{(L / 2+1)^{p}}\right. \\
& \left.-\frac{1}{(L / 2+1)^{p}}+\frac{1}{(L / 2)^{p}}+\ldots-\frac{1}{(L-1)^{p}}+\frac{1}{2^{p}}-\frac{1}{L^{p}}+1\right) .
\end{aligned}
$$

In the ground state and at the ILD transition the en-

ergies $E_{p w}$ and $E_{w e t}$ are equal. For a particular $L$ this 
determines the magnitude $h_{1 w}^{I L D}(L)$ :

$$
\begin{aligned}
h_{1 w}^{I L D}(L) & =1 /\left(1+\frac{1}{2^{p}}+\frac{1}{3^{p}}+\ldots+\frac{1}{(L / 2)^{p}}-\frac{1}{(L / 2+1)^{p}}-\ldots-\frac{1}{(L-1)^{p}}-\frac{1}{L^{p}}\right) \\
& =\left\{\sum_{n=0}^{\infty} \frac{1}{n^{p}}-2 \sum_{n=L / 2+1}^{\infty} \frac{1}{n^{p}}+\sum_{n=L+1}^{\infty} \frac{1}{n^{p}}\right\}^{-1} .
\end{aligned}
$$

In order to find the critical wetting field $h_{1 w}(L \rightarrow \infty)$ we take the limit $L \rightarrow \infty$. According to the second line in Eq. (18) this leads to

$$
h_{1 w}=1 / \sum_{n=0}^{\infty} \frac{1}{n^{p}}=1 / \zeta(p)
$$

where $\zeta(p)$ is the Riemann zeta function. Its values are known analytically only for certain even values: $\zeta(p=$ $2)=\pi^{2} / 6, \zeta(p=4)=\pi^{4} / 90$ which gives $h_{1 w}(p=2) \approx$ 0.6079 and $h_{1 w}(p=4) \approx 0.9239$. Other values can be found in tables of special functions, e.g., $\zeta(p=3) \approx$ 1.2021 giving $h_{1 w}(p=3) \approx 0.8319$. In the short-ranged limit $(p \rightarrow \infty)$ the Riemann zeta function approaches 1 , confirming Abraham's solution at $T=0$ [43]. Even more interesting is the opposite limit $\zeta(p \rightarrow 1) \rightarrow \infty$ which results in $h_{1 w}(p \rightarrow 1) \rightarrow 0$. This means that at $T=0$ the wetting transition does not exist for $p \leq 1$.

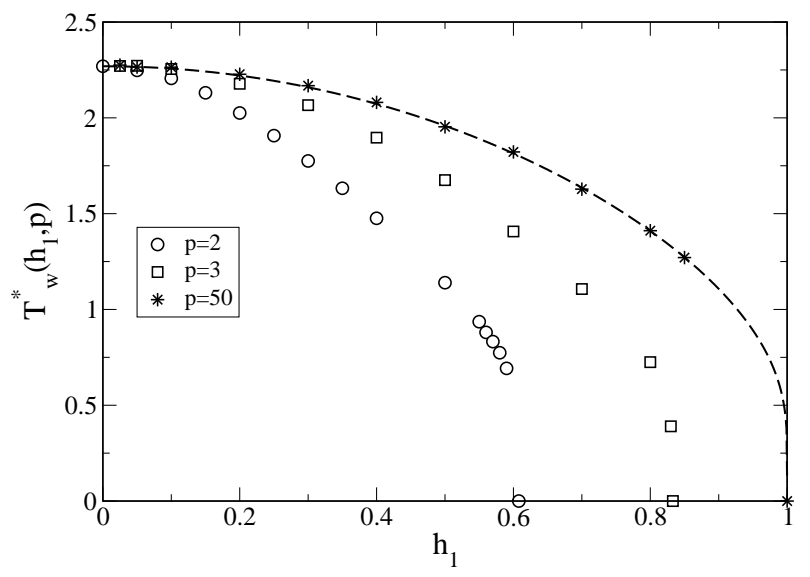

FIG. 5: Phase diagram at bulk coexistence $H=0$ for continuous wetting transitions in the $d=2$ Ising ferromagnet for (Eq. (13) ) $p=2, p=3$, and $p=50$ (quasi-short-ranged boundary field) obtained within the DMRG approach. For $p>1$ the values in the limit $T \rightarrow 0$ are known exactly for semi-infinite systems. The dashed line is the analytically known exact result for $p=\infty$ and for semi-infinite systems [43]. $T_{w}^{*}=k_{B} T_{w} / J$.

In order to determine the location of a quasi-ILD tran- sition between $T=0$ and $T=T_{c}$ for $p<3$ various criteria can be applied [39]. Here we have adopted the approach involving the magnetic susceptibility $\chi$. The singularity (or a maximum) of the magnetic susceptibility $\chi$ is one of the most useful criteria for the localization of a phase transition (or of a pseudo-phase transition for a finite system). The magnetic susceptibility can be calculated as the second derivative of the free energy $f$ with respect to the bulk magnetic field $H$. This method is very convenient for the DMRG approach because the latter provides the free energy with a very high accuracy. Nevertheless the present case is somewhat special, because we want to determine $T_{w}\left(p, h_{1}\right)$ at $H=0$ (see Fig. 5), where in the partial wetting regime, i.e., for $T<T_{w}$, there is a first-order bulk transition. In the thermodynamic limit there is coexistence of phases with opposite magnetizations. Thus there is a discontinuity of the first derivative of the free energy $f$ (a jump of the magnetization $m=-\partial f / \partial H$ ) upon changing the sign of the bulk magnetic field. Accordingly, in order to calculate $\chi$ there, one has to calculate the derivatives for small nonzero bulk fields and then to consider the limit $H \rightarrow 0$. In the complete wetting regime, i.e., $T>T_{w}$, or equivalently above the ILD transition, the finite system exhibits only a single phase with an interface meandering freely between the walls so that there is no discontinuity of the free energy derivatives upon crossing $H=0$. For numerical calculations such as the ones within the DMRG method the necessity of performing an extra limiting procedure ( $H \rightarrow 0$ in this case) is cumbersome. Therefore, instead of $\chi$, we have focused on another quantity $\chi_{0}$, which also corresponds to the second derivative of the free energy at fixed $T$ and $h_{1}$, but is calculated numerically in a symmetrical way with respect to $H=0$ by taking the free energy values at five equidistance points: $-2 \Delta H,-\Delta H$, $0, \Delta H$ or $2 \Delta H$; we typically used $\Delta H=10^{-5}$. Because our calculations are always carried out for finite $L$, there is no discontinuity of the magnetization in the partial wetting regime. These discontinuities are replaced by functions which are rounded but steeply varying at $H=0$. In order to determine the ILD transition we have scanned the phase diagram at fixed $h_{1}$. The higher the temperature, the less steeply the magnetizations vary and the values of their derivative $\chi_{0}$ are smaller. Above the wetting temperature, where there is no discontinuity, 
$\chi_{0}(H=0, T)$ saturates for increasing $T$; here $\chi_{0}$ is equivalent to $\chi$. Therefore, at fixed $L$, the ILD transition can be identified by the maximal slope of $\chi_{0}$ or the minimum of its derivative with respect to temperature. Although all derivatives have been performed numerically, the high accuracy of the DMRG method guarantees very precise results.
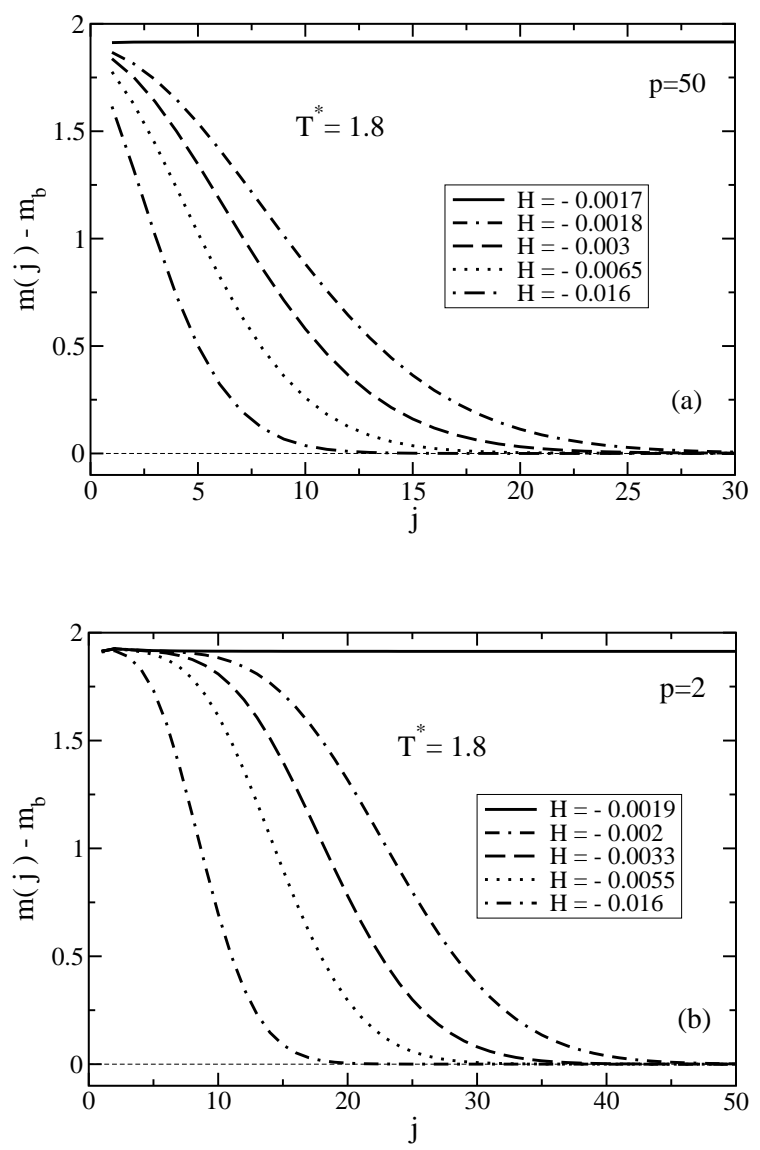

FIG. 6: Magnetization profiles, relative to their bulk values $m_{b}(T, H)<0$, near one wall $(j>1)$ along the isotherm $T^{*}=1.8$ (see path (1) in Fig. 3), calculated within the DMRG method for $d=2$ Ising strips of width $L=500$, for (a) shortranged $(p=50)$ and (b) for long-ranged $(p=2)$ boundary fields of strength $h_{1}=0.8$. Along this isotherm the pseudocapillary condensation transition occurs at $H_{c a} \simeq-0.00172$ for $p=50$ and at $H_{c a} \simeq-0.00195$ (4) for $p=2$. Thus both in (a) and (b) the full line for $H=-0.0017$ and $H=-0.0019$, respectively, corresponds to the capillary filled state with $m(j)-m_{b}(T, H) \simeq 2\left|m_{b}(T, H)\right|$.

Finally we have extrapolated $T_{w}\left(p, h_{1} ; L\right)$ to the limit $L \rightarrow \infty$ in order to obtain the wetting temperatures $T_{w}\left(p, h_{1}\right)$. The obtained wetting phase diagram in the $\left(T, h_{1}\right)$ plane is shown in Fig. 5 for $p=2,3$, and $p=50$. The dashed line is the exact result by Abraham for $p=\infty$ [43]. The close agreement of our data for $p=50$ with this exact result generates confidence in our numerical procedures. Our results show that contrary to the claim by
Kroll and Lipowsky [37] also for $p=2$ there are wetting transitions and that the wetting temperature of these continuous transitions can be well localized as a function of $h_{1}$. In view of the calculations of the adsorption, for our choice $h_{1}=0.8$ the surface is wetted for all temperatures. As already mentioned above, the claim by Kroll and Lipowsky is based on using a continuum planar solidon-solid (SOS) approximation. SOS models ignore bulklike fluctuations such as bubbles and the formation of "overhangs" of the line separating oppositely magnetized regions, while focusing on describing the long-wavelength behavior of the interface, expected to play the crucial role in wetting phenomena. In the present case of the $d=2$ Ising model the bulk fluctuations are particularly strong giving rise to a very diffuse interface. For the case of $p=2$ they might provide the mechanism for the unbinding of the interface from the wall, even though the longwavelength interfacial fluctuations are strongly supressed by this very long-ranged boundary field.

\section{NUMERICAL RESULTS AND DISCUSSION}

Using the DMRG method we have performed calculations of the magnetization profiles $m(j)$ from which we have obtained the thicknesses $\ell_{0}$ of the wetting layer and the adsorption $\Gamma$. The analysis of the shapes of the profiles $m(j)$ and of the scaling properties of $\ell_{0}$ gives a better understanding of the behavior of $\Gamma$ along different thermodynamic paths. We recall, that the predictions for the scaling behavior of the adsorption follows from the scaling behavior of $\ell_{0}$.

In order to infer possible power laws governing the behavior of the thickness of the wetting layer and of the adsorption along different paths we have calculated local exponents of the quantities of interest as a function of $H$ or $\tau$. They are defined as

$$
z_{i}=\left|\frac{\ln Q(i+1)-\ln Q(i)}{\ln x_{i+1}-\ln x_{i}}\right|,
$$

which is the discrete derivative of data $Q$ as a function of $x$ in a log-log plot; here $Q=\ell_{0}$ or $\Gamma$ and $x=H$ or $\tau$. Such a quantity probes the local slope at a given value $x_{i}$ of $x$ at a point $i$ along the path considered. It provides a better estimate of the leading exponent than a log-log plot itself. We have chosen the convention that if $Q$ decays algebraically as a function of $x$ then $Q \sim x^{-z}$ with $z>0$. The high quality of the DMRG data allows us to reliably carry out this numerical differentiation.

\section{A. Isotherms}

\section{Magnetization profiles}

In Figs. 6 and 7 we show a selection of magnetization profiles calculated for a strip width $L=500$ with $p=50$ 
and 2 along the two isotherms $T^{*}=1.8$ and $T^{*}=2.25$ indicated in Fig. 3 as path (1) and (3), respectively.
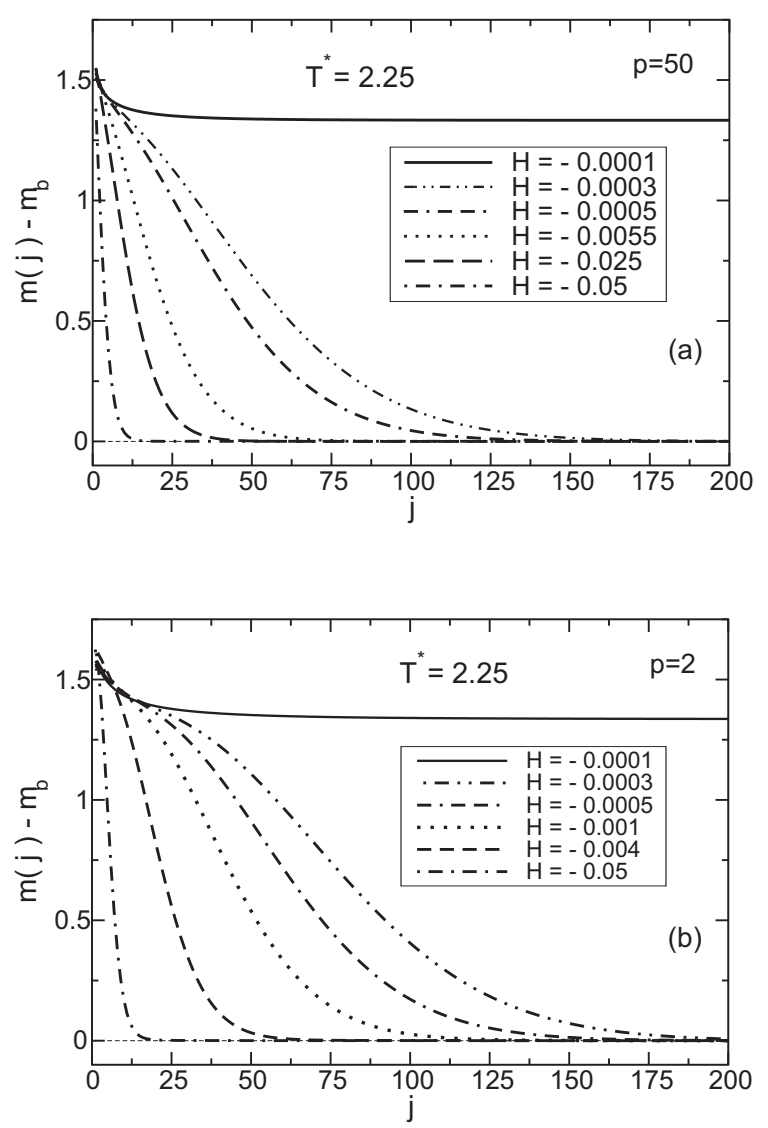

FIG. 7: Magnetization profiles, relative to their bulk values $m_{b}(T, H)<0$, near one wall along the isotherm $T^{*}=2.25$ (see path (3) in Fig. 3), calculated within the DMRG method for $d=2$ Ising strips of width $L=500$, (a) for short-ranged $(p=50)$ and (b) for long-ranged $(p=2)$ boundary fields of strength $h_{1}=0.8$. Along this isotherm the pseudo-capillary condensation transition occurs at $H_{c a} \simeq-0.000191$ for $p=50$ and at $H_{c a} \simeq-0.00026(4)$ for $p=2$. Thus both in (a) and (b) the full line for $H=-0.0001$ corresponds to the capillary filled state with $m(j)-m_{b}(T, H) \simeq 2\left|m_{b}(T, H)\right|$.

From these plots one can see that within the accessible range of values $H<H_{c a}(T, L ; p)$ the shapes of the profiles are not slab-like, even though the system size is very big so that $\left|H_{c a}(T, L)\right|$ is expected to be sufficiently small. They are characterized by a pronounced spatial variation at the emerging interface between the spin up and the spin down phase, where the profile varies quasi linearly with the distance from the wall, and by extended tails. For the short-ranged case $(p=50)$ the profiles along the low-temperature isotherm $\left(T^{*}=1.8\right)$ exhibit a rapid decay to their bulk values $m_{b} \equiv m_{b}(T, H)(<0$ for $H<0)$ with the bulk correlation length $\xi(T, H)=|\tau|^{-\nu} \Xi\left(H|\tau|^{\Delta}\right)$ (see Eqs. (9)-(11)) as decay length which increases upon approaching the pseudo-phase coexistence. Only very close to the pseudophase coexistence line $H_{c a}(T, L ; p)$ the emerging shape of the profile to a certain extent resembles that characteristic of the free interface between the spin up and the spin down bulk phases with a broad interfacial region and a rather narrow region where the magnetization stays somewhat close to the value $-m_{b}>0$ characteristic for the wetting phase corresponding to $H=0^{+}$; this latter region thickens as $H \rightarrow H_{c a}(T, L ; p)$.

For the long-ranged forces $(p=2)$ the profiles along the low-temperature isotherm $\left(T^{*}=1.8\right)$ exhibit an interface-like shape already away from the pseudo-phase coexistence line $H_{c a}(T, L ; p)$. The interfacial part of these profiles is much more pronounced than for the shortranged case $(p=50)$ and their decay is much slower. The exponential decay of the profiles to the value $m_{b}(T, H)$ ultimately crosses over to the power law decay $\sim j^{-2}$. In general, algebraically decaying inter-particle and surface fields are known to generate algebraically decaying order parameter profiles [44].

For both $p=50$ and $p=2$ the profiles corresponding to $H \rightarrow 0$ along the high-temperature isotherm $\left(T^{*}=2.25\right)$ decay much slower as compared to the low-temperature isotherm $\left(T^{*}=1.8\right)$ and their shapes become quantitatively different (see Fig. 7). The magnetization in the first few layers near the walls decreases more rapidly so that there the profiles acquire a positive curvature. Further away from the wall there is an inflection point and the narrow pleateau occurring near the boundaries for the low-temperature isotherm (see Fig. 6(b)) disappears. The interfacial region becomes very broad.

\section{Thickness of the wetting layer}

In order to infer equilibrium thicknesses $\ell_{0}$ of the wetting layers from the magnetization profiles we have chosen the criterion of the change of sign of the curvature of the profile, i.e., we have assigned a thickness to a wetting layer which corresponds to the distance $j=\ell_{0}$ at which the profile exhibits its inflection point. Adopting other criteria for determining the wetting film thickness, e.g., the thickness which corresponds to the distance $j=j_{0}$ at which the magnetization vanishes, $m\left(j_{0}\right)=0$, leads, as it must be, to the same conclusions about physical observables.

We have studied the behavior of $\ell_{0}$ along several isotherms. Representative data are shown and discussed below. They correspond to the isotherms $T^{*}=1.8,2.25$ (see the paths (1) and (3), respectively, in Fig. 3) and $T=T_{c}$ (see path (4) in Fig. 3) and have been obtained for several strip widths and ranges $p=50,5,4,3,2,1.5$ of the boundary fields. (The data for the isotherms $T^{*}=1.6$, 1.9, 2 , and 2.2 are not shown.) We have chosen $h_{1}=0.8$ for which $T_{w}^{*}(p=\infty) \simeq 1$.41. All considered isotherms lie above the corresponding wetting temperatures $T_{w}(p)$.

Along the isotherm $T^{*}=1.6$ the wetting layers are so thin that it is difficult to assign a suitable thickness $\ell_{0}$, especially for $p=50$. Along the isotherm at the higher temperature $T^{*}=1.8$ (path (1) in Fig. 3) this assignment 
is much clearer and the results for $\ell_{0}(H)$ and its local exponents are shown in Fig. 8. They were obtained for strips of width $L=500$ with $h_{1}=0.8$ and for various ranges $p$ of the boundary fields.
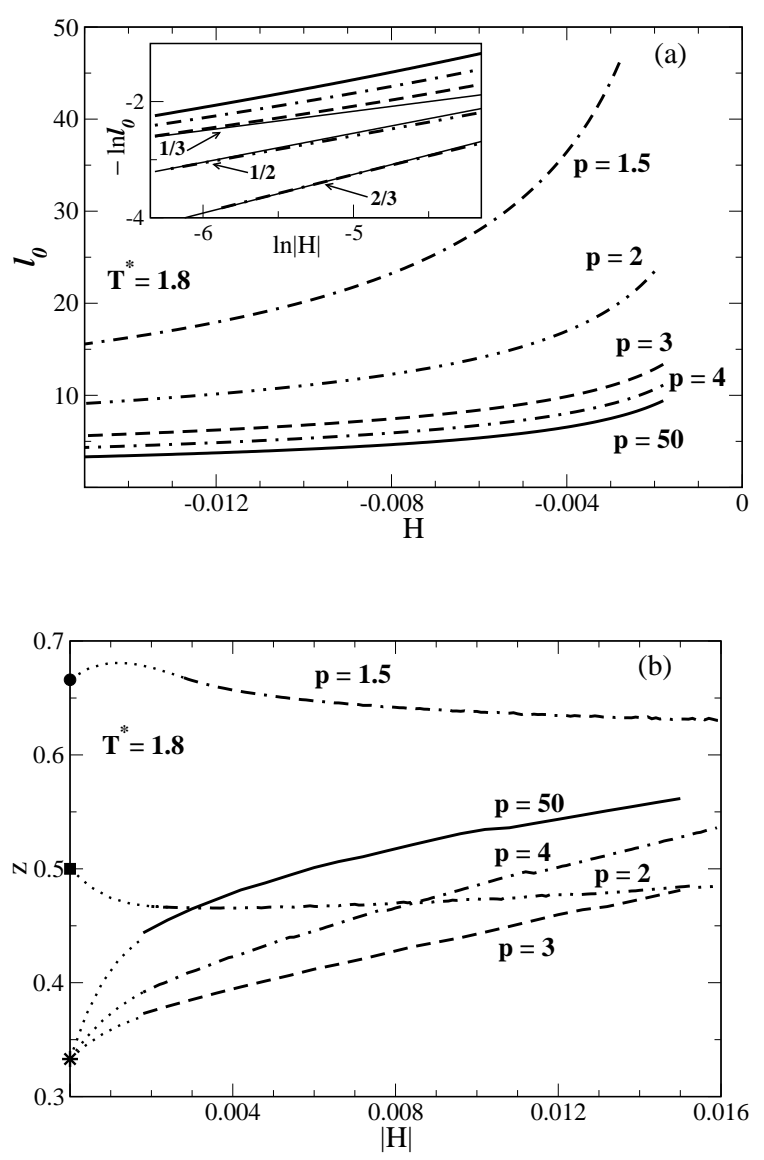

FIG. 8: (a) Equilibrium wetting layer thickness $\ell_{0}$ in units of the lattice constant $a$ as a function of the bulk magnetic field $H$ calculated along the isotherm $T^{*}=1.8$ for $L=500$, $h_{1}=0.8$ and for the ranges $p=1.5,2,3,4$, and 50 of the boundary fields. A rapid increase of $\ell_{0}$ upon approaching the pseudo-phase coexistence line $H_{c a}(T, L ; p)$ supports the existence of the wetting transition for $p=2$ and 1.5. The values of $H_{c a}(T, L ; p)$ are given by the points at which the various lines end on the right. The inset shows a log-log plot of these data. The thin solid lines indicate slopes (from top to bottom): $2 / 3,1 / 2$, and $1 / 3$. (b) The local exponents $z$ for $\ell_{0}(H)$ (see Eq. (20)). For $H \rightarrow 0$, without the occurrence of capillary condensation, i.e., for $L \rightarrow \infty$, the expected exponents are $\beta_{s}^{c o}=1 / 3(\star)$ for $p=3,4$, and $50,1 / 2(\boldsymbol{\square})$ for $p=2$, and $2 / 3(\bullet)$ for $p=1.5$. The dotted lines indicate the expected extrapolations $H \rightarrow 0$ for $L \rightarrow \infty$. The log-log plot supports these conclusions. We note that the local exponents do not always follow from naive visual impressions. For example, $z(p=50)>z(p=2,3,4)$, although in (a) the curves of $\ell_{0}(p=2,3,4)$ seem to increase somewhat stronger than the one for $\ell_{0}(p=50)$.

Path (1) lies entirely within the region between the bulk coexistence curve and the crossover boundary
$H_{c r}^{(1)}=-d_{1}|\tau|^{3 \nu}$ with $\nu=1$ for the $d=2$ Ising model, i.e., within the complete wetting regime (see Fig. 3). Indeed, for all values of $p$ we observe the increase of $\ell_{0}$ upon approaching the pseudo-phase coexistence line $H_{c a}(T, L ; p)$. The lines in Figs. 8, [13 end on the right when they reach the pseudo-phase coexistence line $H_{c a}(T, L ; p)$ (see Fig. 3). Apart from this size dependence of the $H$-window with access for complete wetting, for $L=500$ and 300 (data for $L=300$ are not shown) the finite-size effects are negligible. As expected from the shape of the profiles (see Figs. 6 and 7) for the same value of $H$ the wetting layer is much thicker for small values of the exponent $p$ than for short-ranged surface fields $(p=50)$. The rate of increase of $\ell_{0}$, as characterized by the behavior of the local exponents $z$, depends on the range of the boundary field $p$ and varies along the isotherm. For $p=1.5$ the rate of increase is always largest, i.e., $z$ remains the largest along the entire isotherm (see Fig. $8(\mathrm{~b})$ ). For $p=50,4$, and 3 the local exponent $z$ decreases as $|H| \rightarrow 0$, i.e., the initial increase of the wetting layer is larger than the one observed very close to $H=0$ for which the plateau in the profile near the wall corresponding to the wetting phase at $H=0^{+}$ starts to form. For $p=2$ the very long-ranged nature of the boundary field enforces the occurrence of a narrow plateau in the profile near the wall even far away from $H=0$. Upon decreasing $|H|$ the width of this plateau increases and the emerging interfacial region broadens giving rise to the increase of $\ell_{0}$ which is initially smaller than for $p=50$. Only very close to $H=0$ this trend reverses and eventually the local exponents $z$ corresponding to $p=2$ become larger. For $p=3$ and 4 the rate of increase of $\ell_{0}$ remains smaller than for $p=2$ and 50 along the whole isotherm (for $|H|<0.008$ ). According to the predictions summarized in Sec. II for semi-infinite systems $(L \rightarrow \infty)$, upon approaching the bulk coexistence line $H=0$ the thickness of the wetting film is expected to diverge for $p \geq 3$ with the exponent $\beta_{s}^{c o}=1 / 3$ and for $p<3$ with the exponent $\beta_{s}^{c o}=1 / p$. As can be inferred from Fig. 8 the local exponents $z$ calculated for $p=50,4,3$, and 2 tend towards their predicted values for $H \rightarrow 0$ but cannot reach them due to capillary condensation. For $p=1.5$ the local exponents have to behave non-monotonically in order to reach the expected value $1 / p=2 / 3$. The behavior of $\ell_{0}$ along the isotherm $T^{*}=1.9$ is very similar (data not shown).

Path (2) corresponds to $T^{*}=2.0$ and runs in between the crossover lines given by $H_{c r}^{(1)}=-d_{1}|\tau|^{3 \nu}$ and $H_{c r}^{(2)}=-d_{2}|\tau|^{\Delta}$ with $\nu=1$ and $\Delta=15 / 8$ in $d=2$; it hits the pseudo-phase coexistence line of capillary condensation before it reaches the crossover boundary $H_{c r}^{(1)}=-d_{1}|\tau|^{3}$. Along that path the variation of $\ell_{0}$ is very similar to the one observed along the isotherm $T^{*}=1.8$ with the local exponents approaching the expected values of the complete wetting regime (data not shown). Again the asymptotic behavior is preempted by capillary condensation. 

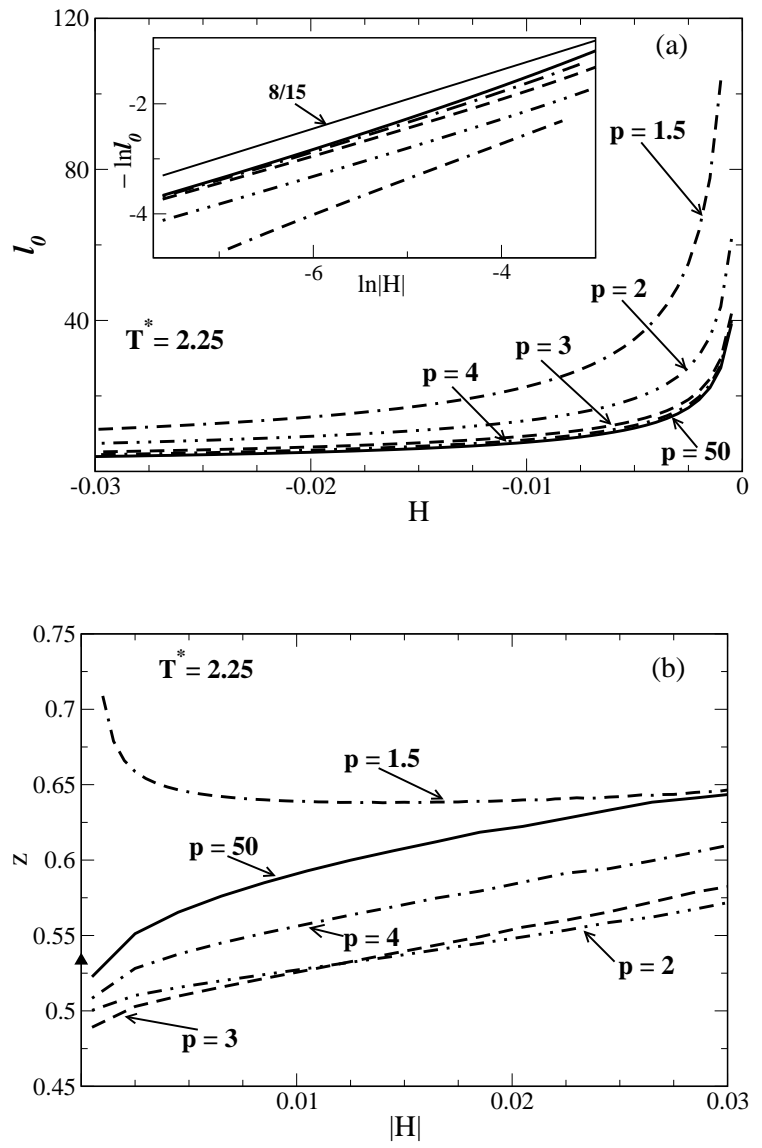

FIG. 9: (a) Wetting layer thickness $\ell_{0}$ in units of the lattice constant $a$ as a function of the bulk magnetic field $H$ calculated along the isotherm $T^{*}=2.25$ (path (3) in Fig. 3) for $L=500, h_{1}=0.8$, and for the ranges $p=1.5,2,3,4$, and 50 of the boundary fields. On the right the curves end at the corresponding capillary condensation point $H_{c a}(T, L ; p)$. The inset shows a log-log plot of these data. The thin solid line indicates the slope 8/15. (b) The local exponents $z$ for $\ell_{0}(H)$ (see Eq. (20). For $p \geq 2$ and $H \rightarrow 0$ they tend towards $0.48, \ldots$, 0.52 whereas the expected value $8 / 15(\boldsymbol{\Delta})$ is somewhat higher. According to Fig. 3] along this isotherm capillary condensation prevents one to reach the ultimate complete wetting regime. For $p=1.5$ the behaviors of $\ell_{0}(H)$ and $z(H \rightarrow 0)$ are distinctly different from those for $p \geq 2$.

Results along the near-critical $\left(T^{*}=2.25\right)$ and critical $\left(T^{*}=T_{c}^{*}\right)$ isotherms (paths (3) and (4) in Fig. 3) are displayed in Fig. 9] and Fig. 10, respectively, for $L=500$. (For $L=300$ we observe a similar behavior; these data are not shown.) Both paths lie in the critical adsorption regime where one expects $\ell_{0}(H \rightarrow 0) \sim \xi \sim|\Delta H|^{-\nu / \Delta}$ with $\nu / \Delta=8 / 15$ in $d=2$. This universal behavior is expected to hold if the long-ranged boundary field decays sufficiently rapidly, i.e., if $p>(d+2-\eta) / 2$. In this case the long-ranged part of the boundary field is irrelevant in the RG sense with respect to a pure contact surface field [2]. Here $\eta$ is the critical exponent governing the algebraic decay of the two-point correlation function in the bulk and at $T_{c}$. In the present case of the $d=2$ Ising model $\eta(d=2)=1 / 4$ so that for $p>15 / 8$ we expect to observe the power law $\ell_{0} \sim|\Delta H|^{-8 / 15}$.

For $p=1.5$ one has $p<(d+2-\eta) / 2$. To our knowledge so far this case has not been studied in the literature in the context of critical adsorption. According to general scaling arguments [2] the magnetization profile for the semi-infinite system under rescaling of distances by a factor $b$ behave as

$$
\begin{aligned}
m_{ \pm}\left(y, \tau, H, h_{1}, p\right)= & \\
& b^{-\beta / \nu} m_{ \pm}\left(y b^{-1}, \tau b^{1 / \nu}, H b^{\Delta / \nu}, h_{1} b^{\omega_{s}}\right),
\end{aligned}
$$

where $\omega_{s} \equiv(d+2-\eta) / 2-p$. Because in the present case $\omega_{s}>0$ the range of such wall-fluid interactions is relevant in the $\mathrm{RG}$ sense at and near $T_{c}$, i.e., the profiles are affected by the decay of the boundary field even if $y$ is sufficiently large; in the critical region the profiles are no longer equivalent to those generated by a pure contact surface field $(p=\infty)$. Choosing $b \sim y$ one obtains the scaling form of the magnetization

$$
m_{ \pm}\left(y, \tau, H, h_{1}, p\right)=y^{-\beta / \nu} \mathcal{M}_{ \pm}\left(y / \xi_{\tau}, y / \xi_{H}, y^{\omega_{s}} h_{1}\right)
$$

where $\xi_{\tau} \equiv \xi_{ \pm}(\tau, 0)$ is given by Eq. (10) whereas $\xi_{H} \equiv$ $\xi(0, \Delta \mu)$ is given by Eq. (11). For $\omega_{s}<0$ and large enough $y$ the scaling function $\mathcal{M}_{ \pm}$can be expanded in terms of their third argument demonstrating that in this case the long range of the wall-fluid interaction gives rise to contributions to the scaling functions of the magnetization profile which are subleading to the dominant universal $y^{-\beta / \nu}$ behavior valid for $y \lesssim \xi$ and $p=\infty$. Such an expansion cannot be performed for large $y$ if $\omega_{s}>0$ and in order to obtain a prediction in this case for the leading behavior of the magnetization profile and hence the thickness of the wetting layer $\ell_{0}$ and the adsorption, one has to calculate the full generalized scaling functions of the magnetization profiles. For $p<15 / 8$ such an analysis represents a necessary future research goal which, however, is beyond the scope of the present study. Nevertheless, we find it instructive and stimulating to show our numerical results for $p=1.5$ because they are strikingly different from those obtained for values of $p$ satisfying $p>(d+2-\eta) / 2$.

As along the noncritical isotherms $T^{*} \leq 2.25$ we find that also along the isotherms $T^{*}=2.25$ and $T=T_{c}^{*}$ the increase of the wetting film thickness upon approaching $H=0$ is much more stronger for $p=1.5$ than for the other values of $p$. For $|H| \lesssim 0.01$ the local exponents attain $z(p=1.5) \simeq 0.64$, which indicates an algebraic increase of $\ell_{0}$ in this range of $H$ with $\ell_{0} \approx|H|^{-0.64}$. This effective exponent is close to the value $1 / p=2 / 3=$ $0.66(6)$ predicted for the complete wetting behavior for $p=1.5$. Closer to $H=0$ the functional form of the increase changes and $z$ increases strongly.

For $p=50,4$, and 3 the local exponents decrease upon approaching the pseudo-phase coexistence line $H_{c a}(T, L ; p)$. This behavior is similar to the one occurring along the isotherm $T^{*}=1.8$; however, apart from 
the case $p=1.5$ the values of $z$ in Fig. 9(b) are larger than the values in Fig. 8(b), which indicates the stronger increase of the wetting film thickness. For $p=2$ the variation of the local exponents differs from that for the isotherm $T^{*}=1.8$ : here $z$ is a decreasing function of $H$, almost identical to the one for $p=3$ (see Fig. 9(b)).
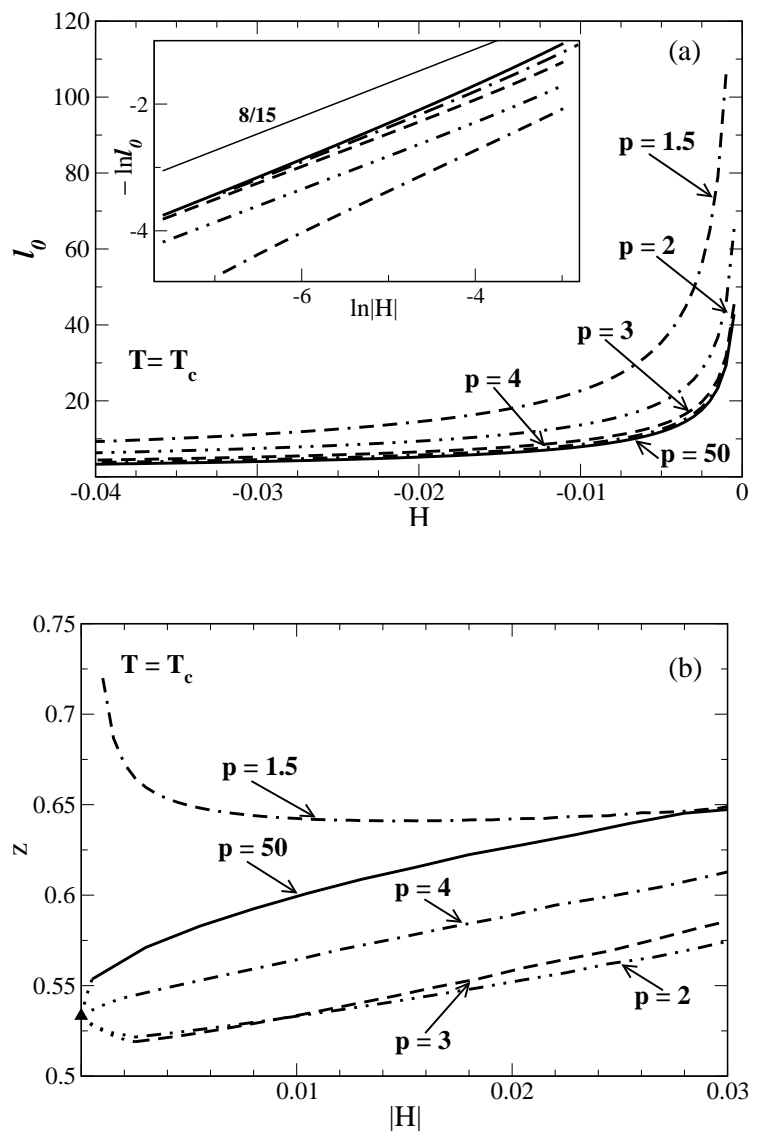

FIG. 10: (a) Wetting layer thickness $\ell_{0}$ in units of the lattice constant $a$ as a function of the bulk magnetic field $H$ calculated along the critical isotherm $T^{*}=T_{c}^{*}=2.269$ (path (4) in Fig. 3) for $L=500, h_{1}=0.8$, and for the ranges $p=1.5,2,3,4$, and 50 of the boundary fields. On the right the curves end at the corresponding capillary condensation point $H_{c a}(T, L ; p)$. The inset shows a log-log plot of these data. The thin solid line indicates the slope $8 / 15$. (b) The local exponents $z$ for $\ell_{0}(H)$ (see Eq. (20)). For $H \rightarrow 0$, without the occurrence of capillary condensation, i.e., for $L \rightarrow \infty$, the expected exponent is $\nu / \Delta=8 / 15(\boldsymbol{\Delta})$. The dotted lines indicate the expected extrapolation for $z(p \geq 2, H \rightarrow 0)$ with $L \rightarrow \infty$. For $p=1.5$ the behaviors of $\ell_{0}(H)$ and $z(H \rightarrow 0)$ are distinctly different from those for $p \geq 2$.

For all decay exponents $p \geq 2$ studied for $T^{*}=2.25$ the values of the local exponents $z(H \rightarrow 0)$ seem to attain values between 0.48 and 0.52 . They would have to bend upwards for $H \rightarrow 0$ in order to reach the expected value of $8 / 15 \simeq 0.533$. For the size $L=500$ capillary condensation does not allow to reach the ultimate complete wetting regime below the crossover line $H_{c r}^{(1)}$ (see
Fig. 3 for path (3)).

For $p=3$ and 2 , the variation of the local exponents $z$ along the critical isotherm $T=T_{c}$ is very similar to the one in Fig. 9(b). In both cases, $z$ first decreases from the value $\approx 0.55$ above the expected one $8 / 15 \simeq 0.53$ to the value $\approx 0.52$ at $|H| \approx 0.001$ and only then, for even smaller values of $H$, they seem to increase towards the expected value 8/15. In the limit $H \rightarrow 0$, for $p=50$ and 4 the local exponents seem to approach the expected value $8 / 15$ from above whereas for $p=3$ and 2 they will have to bend upwards do so from below.
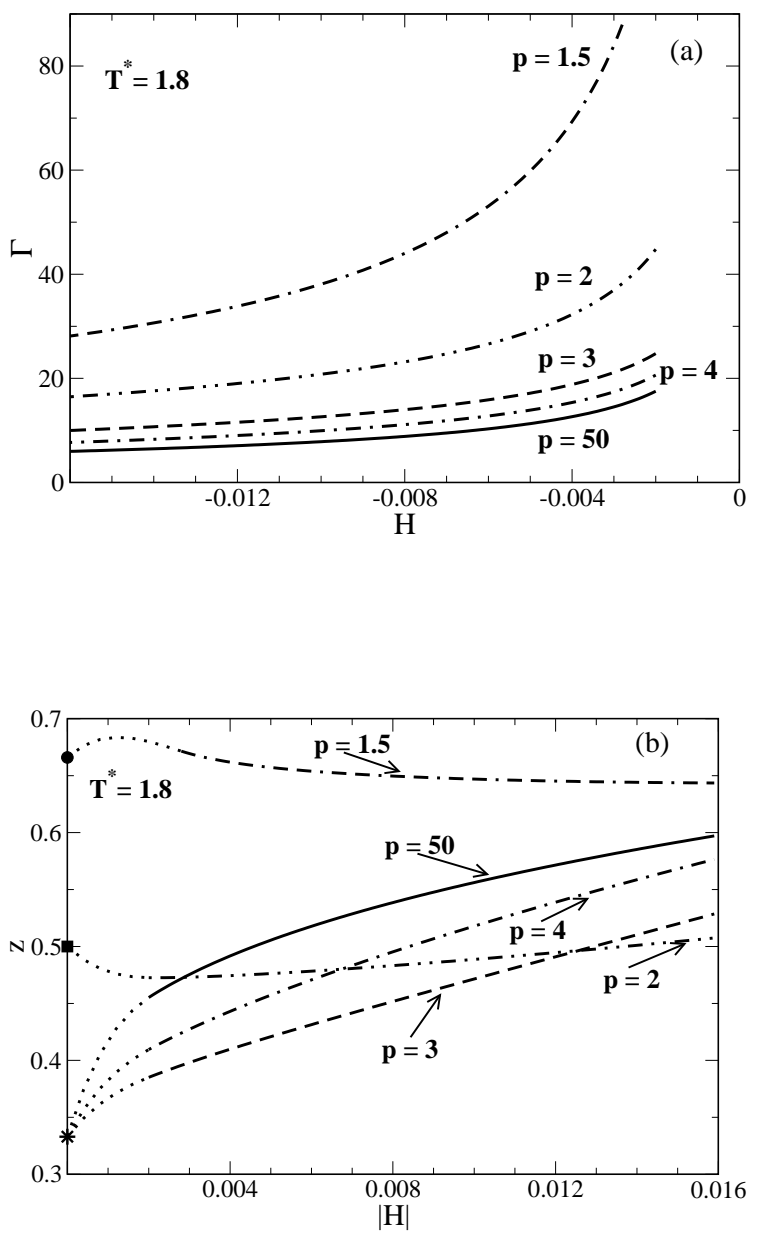

FIG. 11: Adsorption $\Gamma(H)$ (a) and the corresponding local exponents $z$ (b) along the isotherm $T^{*}=1.8$ (path (1) in Fig. 3) calculated within the DMRG approach for a $d=2$ Ising strip of width $L=500$ subject to symmetric boundary fields of strength $h_{1}=0.8$ and various ranges $p$ corresponding to power law decays $\sim j^{-p}$. For small $|H|$ all curves end at $H=H_{c a}(T, L ; p)$ where capillary condensation sets in. Dotted lines indicate the expected extrapolations to $H=0$ for semi-infinite systems: $\beta_{s}^{c o}=1 / 3(\star)$ for $p \geq 3, \beta_{s}^{c o}=1 / 2$ (ם) for $p=2$, and $\beta_{s}^{c o}=2 / 3(\bullet)$ for $p=1.5$. 


\section{Adsorption}

For the present microscopic model in the strip geometry the adsorption $\Gamma$ is defined as

$$
\Gamma=\sum_{j=1}^{L / 2}\left(m_{j}-m_{b}(T, H)\right),
$$

where $m_{j} \equiv\left\langle\sigma_{j}\right\rangle$ is the magnetization in row $j$ and $m_{b}(T, H)$ is the corresponding bulk magnetization. In the limit $L \rightarrow \infty$ this definition reduces to the semiinfinite quantity $\Gamma$ defined in Eq. (3). Along the various paths indicated in Fig. (3), at each point we have first calculated the bulk magnetization $m_{b}(T, H)$ using an equivalent system but with a vanishing surface field in order to minimize the influence of the boundary.
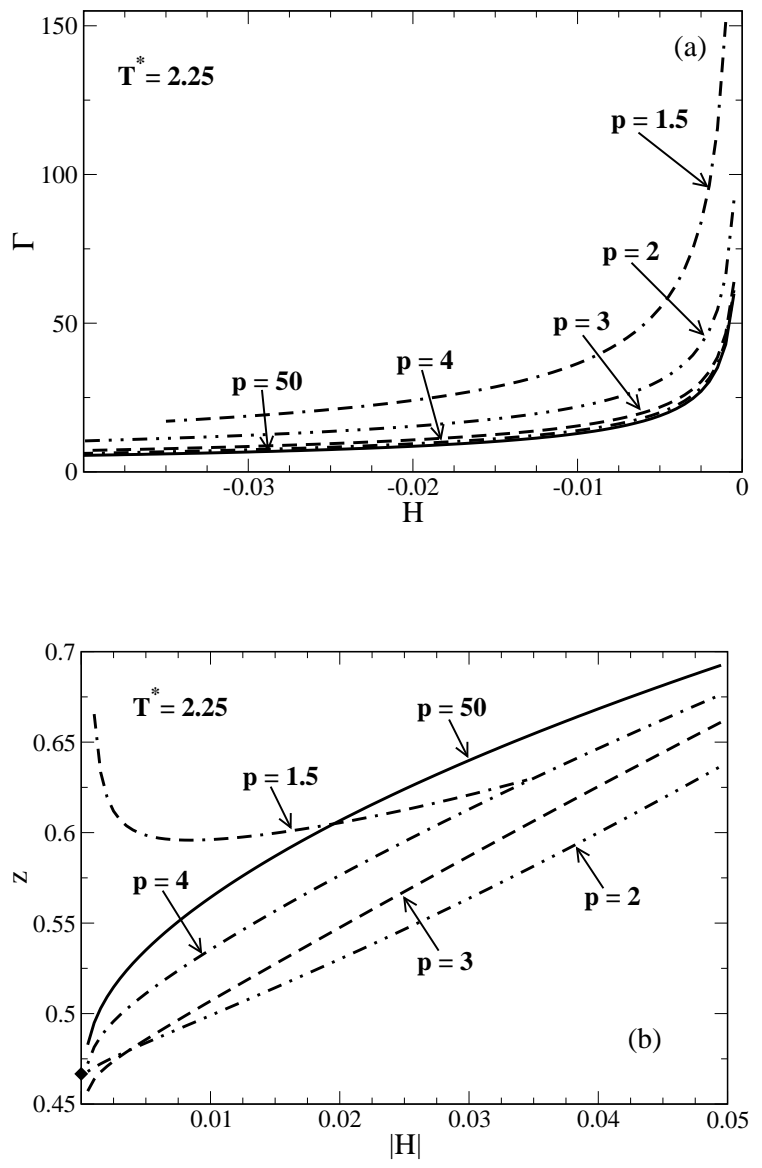

FIG. 12: The same as in Fig. 11]along the isotherm $T^{*}=2.25$ (path (3) in Fig. 3). In (b) $(\nu-\beta) / \Delta=7 / 15$ is indicated (४). The crossover to the ultimate complete wetting behavior is masked by the occurrence of capillary condensation. For $p=1.5$ the behaviors of $\Gamma(H)$ and $z(H \rightarrow 0)$ are distinctly different from those for $p \geq 2$.

Moreover, for the latter calculations as a representative spin the one in the middle of the strip was taken, as it is the one least affected by finite-size effects. In order to obtain the bulk magnetization $m_{b}(T, H)$ we have extrapolated this midpoint value $m_{L / 2}\left(T, H ; h_{1}=0\right)$ to $L \rightarrow \infty$ from data calculated for $L=300,400,500,600$, and 700 .

The adsorption $\Gamma(H)$ and its local exponents calculated for a strip of width $L=500$ with $h_{1}=0.8$ and for various ranges $p$ of the boundary fields are shown in Figs. 11, 12, and 13 for the paths (1), (3), and (4), respectively, as indicated in Fig. 3.
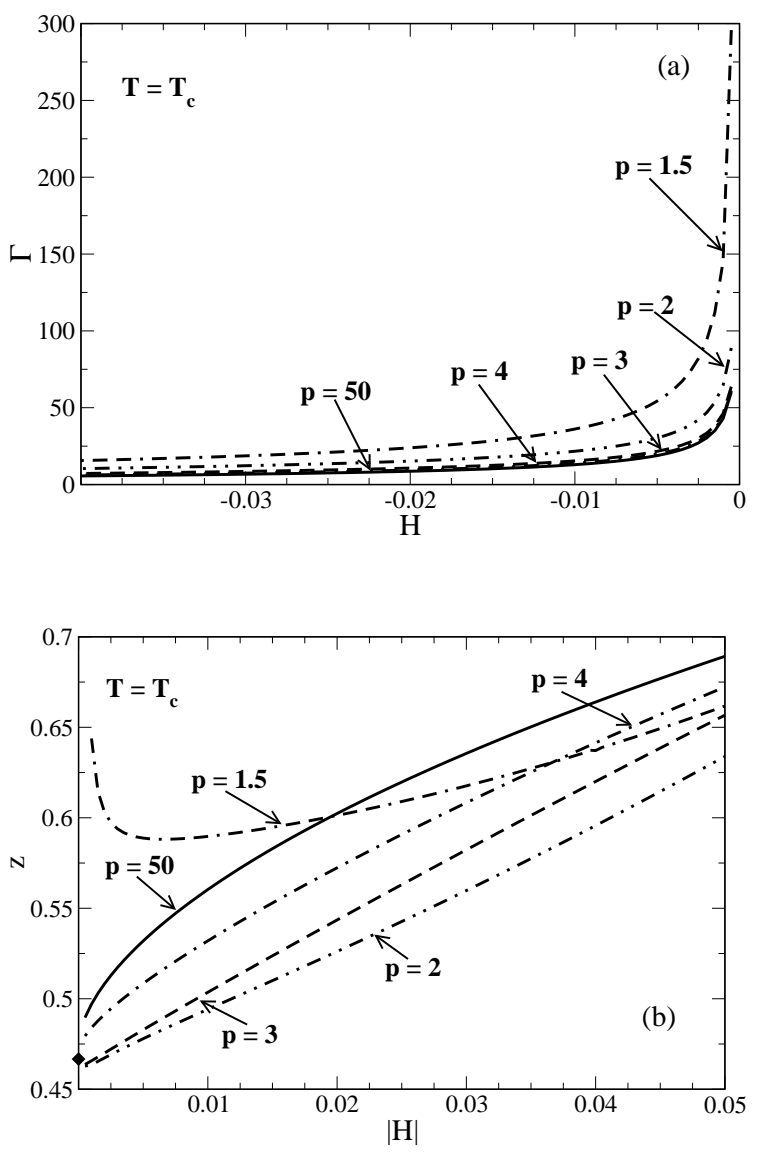

FIG. 13: The same as in Fig. 12 along the critical isotherm $T=T_{c}$ (path (4) in Fig. 3). In (b) $(\nu-\beta) / \Delta=7 / 15$ is indicated $(\checkmark)$. The crossover to the ultimate complete wetting behavior is masked by the occurrence of capillary condensation. For $p=1.5$ the behaviors of $\Gamma(H)$ and $z(H \rightarrow 0)$ are distinctly different from those for $p \geq 2$.

According to the predictions summarized in Sec. II. upon approaching the bulk coexistence line $H=0$ along path $(1)\left(T^{*}=1.8\right)$ the adsorption is expected to diverge for $p \geq 3$ with the exponent $\beta_{s}^{c o}=1 / 3$. As can be seen in Fig. 11(b) the local exponents calculated for $p=$ 50,4 , and 3 tend towards this predicted value for $H \rightarrow 0$ but do not reach it due to the occurrence of capillary condensation. For $p=2$ the local exponents as a function of the bulk field exhibit a negative curvature, consistent with the behavior of $z$ for the thickness $\ell_{0}$ of the wetting 
layer (see previous subsection), and seemingly tend to the exponent $1 / 2$, which agrees with the predicted nonuniversal behavior for $p<3$, i.e., $\beta_{s}^{c o}=1 / p$. In order to reach the expected exponent $\beta_{s}^{c o}=2 / 3$ for $p=1.5$, the local exponents have to vary in a non-monotonic way for $H \rightarrow 0$ (see Fig. 11(b)).

As expected from the analysis of the thickness of the wetting layer, the local exponents for the adsorption $\Gamma(H)$ along path $(2)\left(T^{*}=2.0\right)$ behave in a similar way as along path $(1)\left(T^{*}=1.8\right)$, i.e., for $p=50,4$, and 3 they tend to the complete wetting exponent $\beta_{s}^{c o}=1 / 3$, whereas for $p=2$ to the non-universal value $\beta_{s}^{c o}=1 / p=$ $1 / 2$ (data not shown). As before, the asymptotic behavior is not yet completely reached when capillary condensation occurs. We note that the theoretical considerations in Sec. III do not predict any specific power law behavior in terms of $H$ for the accessible $H$ values along this isotherm (2).

Paths (3) and (4) lie in the critical adsorption regime and one expects (see Eq. (2)) $\Gamma(H \rightarrow 0) \sim|H|^{-(\nu-\beta) / \Delta}$ as $H \rightarrow 0$ for $p \geq 15 / 8$. One can see from Figs. 12(b) and 13(b) that for both $p=50$ and $p=4$ along both isotherms the local exponents seemingly tend to the predicted value $(\nu-\beta) / \Delta=7 / 15$ from above. In order to reach this value for $p=3$ and 2 , the local exponents have to bend upwards for $H \rightarrow 0$; whether for path (3) they ultimately tend to the complete wetting value $\beta_{s}^{c o}=1 / 3$ and $1 / 2$, respectively, after crossing both crossover lines $H_{c r}^{(1)}$ and $H_{c r}^{(2)}$ is unclear because for the finite values of $L$ considered here the asymptotic behavior is undercut by capillary condensation. (Note that for $\beta_{s}^{c o}=1 / 2$ one has $H_{c r}^{(1)}=d_{1}|\tau|^{2 \nu}$.) According to Fig. 3 for $T^{*}=2.25$ this complete wetting regime is expected to be very narrow.

For $p=1.5(<15 / 8)$ the behavior of the critical adsorption is expected to be non-universal. Indeed, as compared to the other values of $p$ we observe a distinctly different behavior of the local exponents along both paths (3) and (4) with $z(H \rightarrow 0)$ strongly increasing. (Note that for $p=1.5$ one has $H_{c r}^{(1)}=d_{1}|\tau|^{3 \nu / 2}$.)

For $\tau \neq 0$ the capillary condensation line $H_{c a}(\tau, L)$ is expected 45] to approach the bulk coexistence line $H=0$ as $1 / L$ for $L \rightarrow \infty$ in accordance with the Kelvin equation (Eq. (14) ). Near $T_{c}$, i.e., for $|\tau| \ll 1$ scaling arguments lead to the scaling behavior (see Eqs. (9) and (10) ):

$$
H_{c a}(\tau, L)=|\tau|^{\Delta} \tilde{g}\left(L / \xi, h_{1}|\tau|^{-\Delta_{1}}\right),
$$

where $\Delta_{1}$ is the surface gap exponent $\left(\Delta_{1}(d=2)=1 / 2\right)$ due to the presence of surface fields [2]; note that $\tilde{g}<0$. (In Eq. (24) the scaling of $h_{1}$ holds if $p$ is sufficiently large, i.e., $p>15 / 8$.) Since $\xi$ depends on $\Delta \mu$ (see Eq. (99), in Eq. (24) the first scaling variable of $\tilde{g}$ depends also on $H_{c a}(\tau, L)$, rendering an implicit equation for $H_{c a}(\tau, L)$. To leading order $H_{c a}(\tau, L)$ is given by Eq. (24) with $\xi$ from Eq. (10). In the following $h_{1}$ is considered to be large enough so that $\tilde{g}(x, u \rightarrow \infty)=g(x)<0$ becomes independent of the second scaling variable. The analytic property $g(x \rightarrow \infty) \sim 1 / x$ renders $H_{c a}(\tau, L \rightarrow \infty) \sim$ $|\tau|^{\Delta-\nu} / L$ for $\tau \neq 0$ fixed and $L \rightarrow \infty$, in accordance with the Kelvin equation. On the other hand, for $L$ fixed and $\tau \rightarrow 0$ one has $g(x \rightarrow 0) \sim x^{-\Delta / \nu}$ (see below), which ensures that the limit $\tau \rightarrow 0$ renders a nontrivial function of $L: H_{c a}(\tau \rightarrow 0, L) \sim L^{-\Delta / \nu}$ with $\Delta / \nu=15 / 8$ in $d=$ 2. For weak surface fields $H_{c a}(\tau, L) \sim h_{1}|\tau|^{\Delta-\Delta_{1}} \bar{g}(L / \xi)$ with $\bar{g}(x \rightarrow 0) \sim x^{-\left(\Delta-\Delta_{1}\right) / \nu}, \bar{g}<0$, so that $H_{c a}(\tau \rightarrow$ $0, L) \sim L^{-\left(\Delta-\Delta_{1}\right) / \nu}$ 46.

The capillary condensation line ends at a capillary critical point, at which the free energy of the confined system expressed in terms of the scaling variables $\left(L / \xi, H|\tau|^{-\Delta}\right)$ is singular at a point $\left(x_{0}, y_{0}\right)$ implying Eq. (15), which is consistent with the scaling behavior $H_{c a}(\tau, L \rightarrow \infty) \sim$ $|\tau|^{\Delta-\nu} / L$. This scaling behavior of the capillary condensation line is sketched in Fig. 2 .

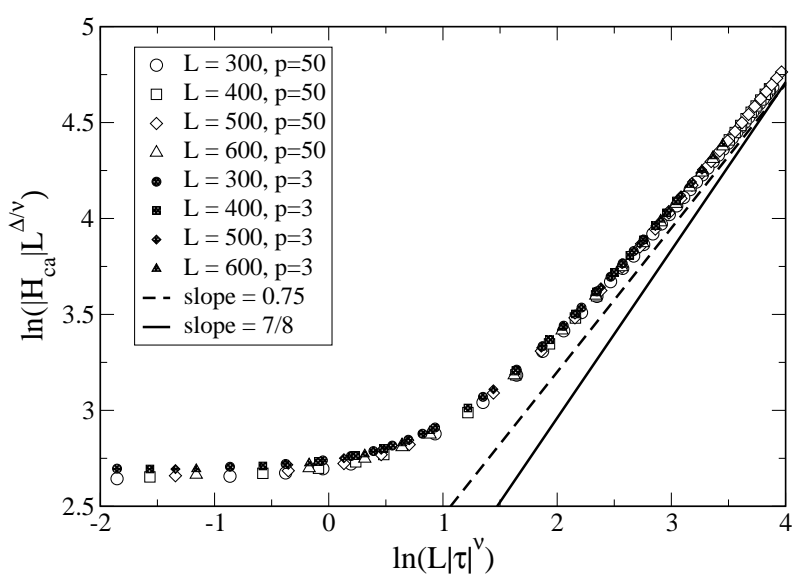

FIG. 14: The log-log plot of the scaling function $\hat{g}(x=L / \xi)$ (see Eq. (25) ) of the capillary condensation line $H_{c a}(\tau, L)$ calculated for several large values of $L$ and for a short-ranged $(p=50)$ and a long-ranged $(p=3)$ boundary field. In the limit $x \equiv L|\tau|^{\nu} \rightarrow 0$ the scaling function saturates at a constant nonzero value whereas $\hat{g}(x \rightarrow \infty) \sim x^{w}$ with $w \simeq 0.75$. The expected value $(\Delta-\nu) / \nu=7 / 8=0.875$ is not yet reached.

With $\xi \sim|\tau|^{-\nu}$ (see Eq. (9)) the scaling form for the capillary condensation line can be written equivalently as

$$
H_{c a}(\tau, L)=L^{-\Delta / \nu} \hat{g}(L / \xi)
$$

where $\hat{g}(x)=x^{\Delta / \nu} g(x)<0$. We test to which extent scaling holds for the present model with $p=50$ and $p=3$ by plotting $\left|H_{c a}\right| L^{\Delta / \nu}$ versus $L|\tau|^{\nu}$ for several strip widths $L$. Although we neglect the $H$ dependence of $\xi$ (see above and compare Eq. (9)) the data collapse is very good. From the log-log plot (see Fig. 14) one can see that $\hat{g}(x \rightarrow 0)=$ const which implies $g(x \rightarrow 0) \sim x^{-\Delta / \nu}$ (see above) and $H_{c a}(0, L) \sim L^{-\Delta / \nu}$. In the limit $x \gg 1$ our data fit very well to a power law $\hat{g}(x \rightarrow \infty) \sim x^{w}$ with the effective exponent $w \simeq 0.75$. $w$ is smaller than the value $(\Delta-\nu) / \nu=7 / 8=0.875$ which would lead to the predicted behavior $H_{c a}(\tau, L \rightarrow \infty) \sim|\tau|^{\Delta-\nu} / L$. One 
might be inclined to put the blame for the fact that $w$ has not yet reached this expected asymptotic value on corrections caused by the aforementioned $H$ dependence of the bulk correlation length, according to which Eq. (25) reads $H_{c a}(\tau, L) L^{\Delta / \nu}=\hat{g}\left(L|\tau|^{\nu}\left(\Xi\left(H_{c a}|\tau|^{-\Delta}\right)^{-1}\right)\right.$ (see Eq. (9) ); this additional dependence actually spoils the scaling in terms of the scaling variable $x=L|\tau|^{\nu}$. Since, however, scaling is observed, the latter dependence must be weak and the too small value of the exponent $w$ must be due to other corrections.
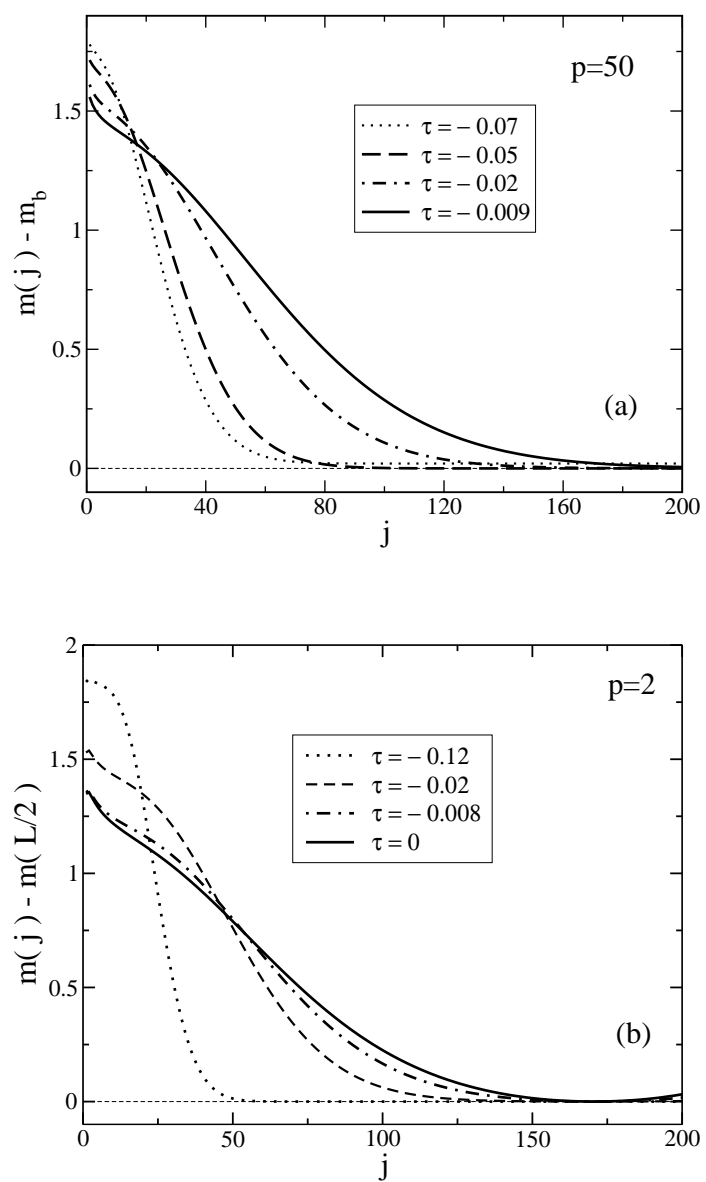

FIG. 15: Magnetization profiles near one wall, relative to their bulk values $m_{b}(T, H)$, along the thermodynamic path (0) (see Fig. 3) shifted by a constant $-10^{-6}$ away from the pseudo-phase coexistence line $H_{c a}(T, L)$, calculated within the DMRG method for $d=2$ Ising strips of width $L=700$ for short-ranged ((a) $p=50)$ and of width $L=340$ for longranged ((b) $p=2)$ boundary fields of strength $h_{1}=0.8$. Due to the slow decay, the profiles for $p=2$ are presented relative to their values $m(L / 2) \neq m_{b}(T, H)$ at the midpoint of the film.

Due to the scaling behavior $H_{c a}(\tau, L) \sim|\tau|^{\Delta-\nu} / L$, for $L \gg 1$ but fixed, the pseudo-capillary condensation line appears to approach the bulk critical point (but without reaching it actually due to $L<\infty)$ as $|\tau|^{\Delta-\nu}=|\tau|^{7 / 8}$ which is a weaker power law than $\left|H_{c r}^{(1)}\right| \sim|\tau|^{\nu / \beta_{s}^{c o}}=|\tau|^{3}$ for the crossover line to complete wetting. This explains the observation that in $d=2$, upon approaching bulk coexistence sufficiently close to the critical point, the asymptotic complete wetting regime is always preempted by capillary condensation. More generally, we expect that in confined systems the asymptotic complete wetting divergence of the adsorption can be observed only if $\Delta-\nu=2-\alpha-\beta-\nu>\nu / \beta_{s}^{c o}$. This condition is fulfilled neither in $d=2$ with $\Delta-\nu=0.875$ and $\nu / \beta_{s}^{c o}=3$ nor in $d=3$ with $\Delta-\nu=0.936$ and $\nu / \beta_{s}^{c o}=3 \nu=1.89$. Thus we conclude that along an isotherm close to $T_{c}$ the ultimate crossover to the complete wetting behavior can only be observed in systems with macroscopically large transverse extensions.

In the present confined system the analogue of the thermodynamic path parallel to the bulk coexistence line on the gas side with a small undersaturation $\Delta \mu=$ const $>0$ and with $T \rightarrow T_{c}$ is the thermodynamic path $H_{(0)}(T, L)=H_{c a}(T, L)+\delta H$ along the pseudo-phase coexistence (capillary condensation) line $H_{c a}(T, L)$ shifted slightly by $\delta H$ to the spin down $(\sigma=-1)$ side (see path $(0)$ in Fig. 3). In view of the large computational effort required for the determination of the curve $H_{c a}(T, L)$ and of the value of the bulk magnetization $m_{b}(T, H)$ at each point along this line we have calculated the adsorption along this path only for a short-ranged boundary field $(p=50)$, for the marginal case $p=3$ of long-ranged surface fields, and for $p=2$. We have considered three constant shifts away from the pseudo-phase coexistence line: $\delta H=-10^{-5},-10^{-6}$, and $-10^{-7}$.

\section{Magnetization profiles}

In Fig. 15 we have plotted a selection of magnetization profiles $m(z)$ along the thermodynamic path shifted by a constant $-10^{-6}$ away from the pseudo-coexistence line $H_{c a}(T, L)$ for $p=50$ (Fig. 15(a)) and for $p=2$ (Fig. 15(b)). For $p=3$ the profiles look qualitatively the same as for $p=2$; they are both characterized by a much broader interfacial region and larger tails than the ones for $p=50$. The shape of the profiles changes upon approaching bulk coexistence $H=0$ in a way similar to the case along the near-critical isotherm $T^{*}=2.25$. One observes a broadening of the interfacial region together with a rather small region where the magnetization stays close to the value $m_{b}(T, H)>0$ characteristic for being slightly on the upper side of the bulk coexistence curve, i.e., $H=0^{+}$.

\section{B. Thermodynamic path along the pseudo - phase coexistence line}

\section{Thickness of the wetting layer.}

For three undersaturations $\delta H$ relative to the pseudophase coexistence line Fig. 16)(a) shows the thickness of 
the wetting layers as a function of temperature calculated for a fixed strip width $L=340$ with strength $h_{1}=0.8$ and ranges $p=50,3,2$ of the boundary fields. The increase of the thickness of the wetting layer along these paths is very similar for all considered values of the decay exponent $p$.
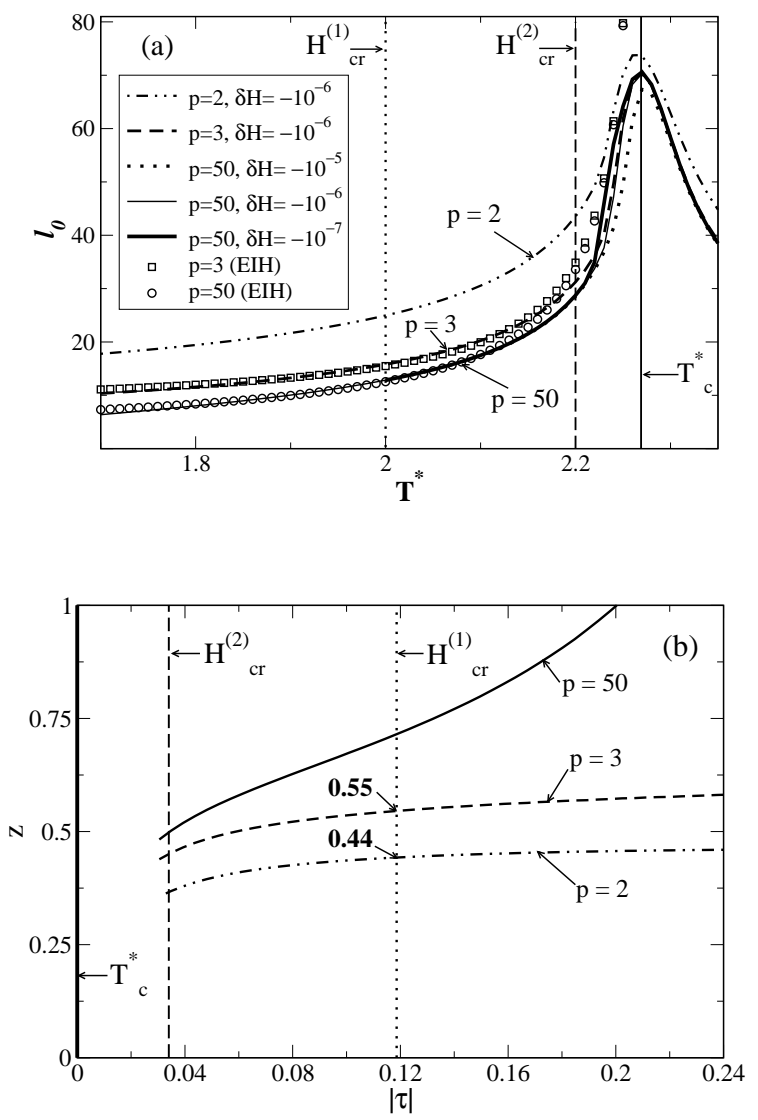

FIG. 16: (a) Wetting layer thickness $\ell_{0}$ (defined as in Figs. 9 and 10) as a function of temperature $T^{*}$ calculated for $L=$ $340, h_{1}=0.8$, and various values of the exponent $p$, characterizing the range of the boundary fields, along the path (0) in Fig. 3 with constant shift $\delta H=-10^{-6}$ away from the pseudo-phase coexisting line $H_{c a}(T, L ; p)$ (see main text). For $p=50$, in addition the temperature dependence of $\ell_{0}$ for shifts $\delta H=-10^{-5}$ and $-10^{-7}$ is shown (dotted and thick solid line, respectively). Symbols denote predictions from the effective interface Hamiltonian (EIH) for $p=50$ (circle) and for $p=3$ (square) (see main text). (b) The local exponents $z$ for $\ell_{0}$ calculated from the data with constant shift $\delta H=-10^{-6}$ shown in (a) as a function of $\tau=\left(T-T_{c}\right) / T_{c}$. At the dotted and dashed vertical line the thermodynamic paths pass through the crossover line $H_{c r}^{(1)}$ and $H_{c r}^{(2)}$, respectively, shown in Fig. 3 (and de facto independent of $\delta H$ ). Due to the finite peak of $\ell_{0}$ at $T_{c}$, below $H_{c r}^{(2)}$, i.e., for $|\tau| \lesssim 0.04$, the concept of the local exponents turns out to be less useful so that the lines in (b) have not been continued into this region.

At low temperatures, for $p=2$ the wetting layer is about three times thicker than for the short-ranged boundary field. Except for the close vicinity of the critical temperature, i.e., for $-0.98 \lesssim T^{*}-T_{c}^{*} \lesssim 1.01$, where the finite-size effects are very pronounced, results obtained along paths with different shifts $\delta H$ but the same $p$ collapse onto a common curve. We note that for $p=50$ this common curve splits into three different lines just above the pseudocritical temperature $T_{c, L}^{*}\left(L=340, h_{1}=0.8\right) \approx 2.2$, i.e., when the pseudocoexistence line $H_{c a}(T, L)$ intersects the crossover line $H_{c r}^{(2)}=-d_{2}|\tau|^{\Delta}$. For each $p$ the thickness of the wetting film attains a certain $L$-dependent value at the bulk critical temperature $T_{c}$, which also depends on the value $\delta H$ of the shift of the path (0). In order to calculate $\ell_{0}\left(T=T_{c}\right)$ for large $L$ we have performed an extrapolation scheme for the curves $\ell_{0}(T)$ to $T=T_{c}$, not taking into account data for temperatures higher than $T^{*}=2.24$ because those exhibit pronounced finite-size effects beyond the leading $L$-behavior. For $p=50$ we have found (data not shown) that the thickness of the wetting layer at $T=T_{c}$ is proportional to $L$, which is expected because $\ell_{0}$ cannot grow larger than $L / 2$. For $p \geq 2$ it should diverge for $L \rightarrow \infty$. Above $T_{c}$ we observe a rapid decrease of $\ell_{0}$ as function of $T$.

For increasing $T \lesssim T_{c}$ in Fig. 16(a) we observe a continuous increase of the thickness of the wetting layer. We recall that, according to effective interface Hamiltonian studies, sufficiently far away from criticality, i.e., for $\ell_{0} \gg$ $\xi$, the wetting layer thickness should be independent of temperature along the path of constant undersaturation $\Delta \mu$, i.e., $H=$ const with respect to bulk coexistence (see the last paragraph of Sec. III). The present results are calculated along $H=H_{(0)}(L, T)=H_{c a}(T, L)+\delta H$ with $\delta H=$ const $<0$ such that $|H|$ decreases for increasing temperatures, which should result in thicker films. This is in accordance with the data in Fig. 16(a). In order to check whether this increase is captured by effective interfacial models we adopt a simple effective interface potential $W(\ell)$ valid for quasi short-ranged $(p=50)$ boundary fields as well as for long-ranged boundary fields with a decay exponent $p=3$ or 2 . Taking an undersaturation $H$ into account, we make the ansatz [3] (in units of $k_{B} T_{c} / a^{2}$ )

$$
W(\ell)=A \ell^{-(p-1)}+B \ell^{-\kappa}+|H| \Delta m \ell
$$

where an effective repulsive interaction $\sim B$, which accounts for the gain in entropy of the unbinding interface, has to be added to the leading long-ranged potential energy term $\sim A>0$ (see Subsec. [IA [26]. One has $B=B_{0}\left|k_{B} T / \Sigma(T)\right|>0$ [26], where $\Sigma(T)$ is the surface stiffness of the interface, $B_{0}=$ const and $\Delta m=2\left|m_{b}^{(0)}(T)\right|$. The exponent $\kappa$ describing thermal wandering is given by $\kappa=2(d-1) /(3-d)$, which equals 2 in $d=2$ (see Subsec. ПA) 26.

In Eq. (26) the second (entropic) term dominates in $d=2$ for $p>3$. Thus for short-ranged forces $(p \gg 1)$ the equilibrium wetting film thickness varies as function 
of $T$ along a thermodynamic path $H(T)$ as

$$
\ell_{0}=\left(B_{0} k_{B} T /\left(\Sigma(T)\left|m_{b}^{(0)}(T)\right|\right)\right)^{1 / 3}|H(T)|^{-1 / 3}, \quad p>3 .
$$

For $p=3$ the entropic term competes with the interaction term. This leads to the equilibrium thickness of the wetting film

$$
\begin{aligned}
& \ell_{0}= \\
& \left(\left(A+B_{0} k_{B} T / \Sigma(T)\right) /\left|m_{b}^{(0)}(T)\right|\right)^{1 / 3}|H(T)|^{-1 / 3}, \quad p=3 .
\end{aligned}
$$

$A$ is proportional to $\Delta m=2\left|m_{b}^{(0)}(T)\right|$ so that $A /\left|m_{b}^{(0)}(T)\right|$ only weakly depends on $T$. For $p=2$ the first term in Eq. (26) dominates and the temperature dependence of the film thickness follows that of $H(T) \simeq H_{c a}(T, L):$

$$
\ell_{0}=\left(A /\left(2\left|m_{b}^{(0)}(T)\right|\right)\right)^{1 / 2}\left|H_{c a}(T, L)\right|^{-1 / 2}, \quad p=2 .
$$

For the $d=2$ Ising model the surface stiffness is known exactly as $\Sigma(T) /\left(k_{B} T\right)=\sinh 2\left(K-K^{*}\right)$ where $K=J /\left(k_{B} T\right)$ and $\tanh K^{*}=\exp (-2 K)$. Both $\Sigma(T)$ and $\Delta m(T) \equiv 2\left|m_{b}^{(0)}(T)\right|$ decrease with increasing temperature. Therefore, for all values of $p, \ell_{0}$ is expected to increase along the pseudo-coexistence line $H_{c a}(T, L)$.

Sufficiently close to the bulk critical point the surface stiffness vanishes with the same power law as the surface tension of the interface $\sigma$, i.e., $\Sigma(\tau \rightarrow 0) \sim$ $\sigma \sim|\tau|^{(d-1) \nu}=|\tau|$ for the $d=2$ Ising model. Since $\Delta m \sim|\tau|^{\beta}$ it follows that in the critical region for shortranged forces

$$
\begin{array}{r}
\ell_{0} \sim|\tau|^{-(\beta+(d-1) \nu) / 3}\left|H_{c a}(\tau, L)\right|^{-1 / 3}= \\
|\tau|^{-3 / 8}\left|H_{c a}(\tau, L)\right|^{-1 / 3}, \quad p=3 .
\end{array}
$$

with $H_{c a}(\tau, L)$ given by Eq. (25).

Close to $T_{c}$ and in the temperature range for which the pseudo-coexistence line $H_{c a}(\tau, L)$ lies in between the crossover lines $H_{c r}^{(1)}=-d_{1}|\tau|^{3 \nu}$ and $H_{c r}^{(2)}=-d_{2}|\tau|^{\Delta}$ we have found (see Fig. 144), for both $p=50$ and $p=3$, that the absolute value of the scaling function $\hat{g}(x=L / \xi)$ of the pseudo-coexistence line (see Eq. 250) decreases and crosses over from the power law behavior $\hat{g}(x \rightarrow \infty) \sim x^{w}$ with $w \simeq 0.75$ for $|\tau| \neq 0$ to a constant value $\hat{g}(x=0)$ at $\tau=0$. This implies that, for decreasing $|\tau|, \ell_{0}(\tau)$ crosses over from an increase $\sim|\tau|^{-5 / 8}$ to one $\sim|\tau|^{-3 / 8}$ (for $L$ fixed). Thus the effective interface model predicts that, along the path $(0), \ell_{0}(\tau \rightarrow 0)$ is an increasing function of temperature. But in view of the aforementioned crossover one cannot expect a purely algebraic behavior in this region.

For $12 \lesssim L|\tau|^{\nu} \lesssim 50$, the variation of the pseudocoexistence line $H_{c a}(T, L)$ at fixed $L$ is approximately algebraic with an effective exponent $w \simeq 0.75$. Inserting $H=H_{c a}(T, L) \sim|\tau|^{0.75}$ into the expression for $\ell_{0}$
(Eq. (30) ) gives $\ell_{0} \sim|\tau|^{-0.625}$ for both $p=50$ and $p=3$. For $L|\tau|^{\nu} \ll 1$ one has $H_{c a} \rightarrow$ const, so that in this limit Eq. (30) predicts $\ell_{0}(\tau \rightarrow 0) \sim|\tau|^{-3 / 8}$.

For $p=2$ the pseudo-coexistence line $H_{c a}(T, L)$ seems to follow a power law with the same effective exponent as for $p=50$ and 3 for reduced temperatures smaller than the crossover line $H_{c r}(1)$ (see Fig. (3). Therefore on the basis of Eq. (29) for $12 \lesssim L|\tau|^{\nu} \lesssim 50$ one expects $\ell_{0} \sim|\tau|^{-0.375}$.

For $p=50$ the behavior of the local exponents $z$ determined from the numerical data obtained for $L=340$ and shown in Fig. [16(a) is consistent with the above predictions (see Fig. 16(b)). We find that in between the crossover lines $H_{c r}^{(1)}=-d_{1}|\tau|^{3 \nu}$ and $H_{c r}^{(2)}=-d_{2}|\tau|^{\Delta}$ the local exponents $z$ decrease continuously upon decreasing $|\tau|$. For $0.12 \lesssim L|\tau|^{\nu} \lesssim 50$, which with $L=340$ and $\nu=1$ corresponds to $0.04 \lesssim|\tau| \lesssim 0.15$, the value of $z$ for $p=50$ changes from $\simeq 0.80$ to $\simeq 0.52$. No saturation at the value 0.625 , which would reflect the power law $\hat{g}(x) \sim x^{0.75}$ (see Eq. (25) and the discussion above), is observed. However, the above temperature range lies outside the range of validity of the asymptotic power law behaviors of $\Sigma(T)$ and $\Delta m(T)$ leading to deviations from the algebraic variation $\ell_{0} \sim|\tau|^{-0.625}$ discussed above.

In order to test the validity of the effective interface Hamiltonian approach we compare our full DMRG data with the predictions for the increase of the wetting film thickness along the line of pseudo-phase coexistence $H_{c a}(T, L)$ stemming from Eq. (27) by substituting our numerical data for $H(T)=H_{c a}(T, L)$ and $\Delta m(T)=2\left|m_{b}(T)\right|$ and by using the analytic expression for $\Sigma /\left(k_{B} T\right)$ given above. Treating $B_{0}$ as a fitting parameter we find very good agreement between these two approaches for $B_{0}=0.729$ (see Fig. 16(a)) in the wide temperature range below $T^{*} \simeq 2.215$. We note, that for higher temperatures the system crosses over to the critical adsorption regime (beyond the crossover line $H_{c r}^{(2)}$ ).

For $p=3$ and 2 we observe that at $H_{c r}^{(1)}$ the local exponents $z$ saturate at the values $\simeq 0.55$ and $\simeq 0.44$, respectively. In the case $p=3$ the exponent is slightly smaller than the prediction 0.625 based on the scaling behavior obtained within the effective interface model, whereas for $p=2$ it is slightly larger than the value 0.375 expected also within the effective interface model.

We repeat the procedure described above to check the agreement of the effective interface Hamiltonian prediction in Eq. (28) with our DMRG data for $p=3$. We adopt $B_{0}=0.729$, i.e., the value determined from the fitting of Eq. (27) to the DMRG results for $p=50$, and treat $A /\left|m_{b}(T)\right| \approx$ const $\equiv C$ as a free parameter. Very good agreement is obtained for $C \approx 1.5$ and temperatures $T^{*} \lesssim 2.15$ (see Fig. [16(a)).

However, the assumption $A /\left|m_{b}(T)\right| \approx$ const does not lead to an agreement of the DMRG data for $p=2$ (not shown) with the curve predicted by Eq. (29) with $H(T)=$ $H_{c a}(T, L)$. Thus we conclude that in the case $p=2$ (not shown) the simple effective interface Hamiltonian given 
by Eq. (26) fails to describe the full DMRG data.

\section{Adsorption}

Results for the adsorption obtained for $L=340, h_{1}=$ 0.8 , and $p=50,3,2$ are shown as a function of $T$ in Fig. 17(a).
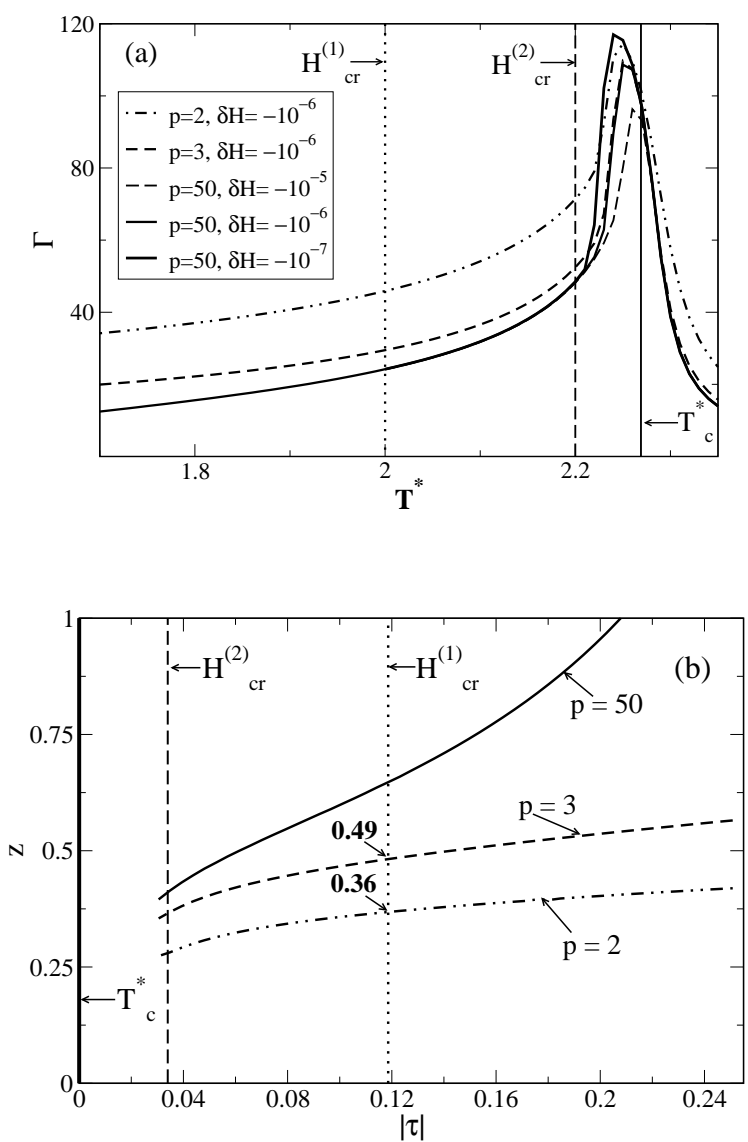

FIG. 17: (a) Adsorption $\Gamma$ as a function of $T^{*}$ along the path (0) in Fig. 3 with a constant shift $\delta H=-10^{-6}$ away from the pseudo-phase coexisting line $H_{c a}(T, L ; p)$ (see main text), calculated within the DMRG method for $d=2$ Ising strips of width $L=340$ for short-ranged $(p=50)$ and longranged $(p=3,2)$ boundary fields of strength $h_{1}=0.8$. For $p=50$ the temperature dependence of $\Gamma$ for shifts $\delta H=$ $-10^{-5}$ and $-10^{-7}$ is also shown (dotted and thick solid line, respectively). (b) The local exponents $z$ for $\Gamma$ calculated from the data shown in (a) for the constant shift $\delta H=-10^{-6}$. At the dotted and dashed vertical line the thermodynamic path passes through the crossover lines $H_{c r}^{(1)}$ and $H_{c r}^{(2)}$, respectively, shown in Fig. 3 (and de facto independent of $\delta H$ ). As in Fig. 16 (b), in (b) the lines do not continue into the region $|\tau| \lesssim 0.04$.

In accordance with the expectations, the behavior of $\Gamma(T)$ upon increasing the temperature is qualitatively similar to the behavior of $\ell_{0}(T)$ in Fig. 16(a). Results for different shifts $\delta H$ form a common curve above $T_{c}$ and for $T^{*} \lesssim 2.2$. Close to $T_{c}$ the adsorption exhibits a maximum which signals the vicinity of the end of the pseudo-phase coexistence line, i.e., the pseudo-critical point of a finite system. The position of this maximum shifts towards $T_{c}$ for wider strips. At $T_{c}, \Gamma$ attains a finite value $\Gamma(\tau=0, L)$. As expected, for long-ranged boundary fields the adsorption is slightly stronger than for short-ranged forces.

According to the discussion in the last paragraph of Sec. III for $p \geq 3$ and in between the two crossover lines $H_{c r}^{(1)}=-d_{1}|\tau|^{3 \nu}$ and $H_{c r}^{(2)}=-d_{2}|\tau|^{\Delta}$ (see Fig. 3) $\Gamma$ is expected to increase $\sim|\tau|^{-(\nu-\beta)}$, which for $d=2$ corresponds to an exponent -0.875 , provided the variation of $\xi$ is dominated by its dependence on $\tau$ (Eq. (10)). This is the case along paths of constant undersaturation $\Delta \mu \equiv H=$ const. Along the pseudo-coexistence line and for $p=50$ the local exponents of $\Gamma$ vary strongly with temperature, also below the crossover line $H_{c r}^{(1)}$ (see Fig. 17(b)). This is not compatible with the aforementioned algebraic variation of $\Gamma$ for $H=$ const. In view of the discussion of the corresponding behavior of the wetting film thickness in the previous subsection, this difference can be traced back to the fact that the pseudocoexistence line does not run in parallel to the bulk coexistence line. The decrease of the absolute value of the bulk field $H_{c a}(T, L)$ upon increasing temperature matters and in the scaling analysis of $\Gamma$ the whole scaling form of $\xi$ (Eq. (9) ) has to be taken into account. According to Eqs. (8) and (9) this is encoded in the product of the scaling functions $\Xi_{ \pm}$and $K_{ \pm}$as function of $H|\tau|^{-\Delta}$, which alters the temperature dependence $\sim|\tau|^{-(\nu-\beta)}$. Due to $\Gamma \sim \ell_{0} \Delta m$ and the fact that $\Delta m$ decreases upon decreasing the reduced temperature $|\tau|$, one expects that the increase of $\Gamma$ is weaker than the increase of $\ell_{0}$. This is consistent with our numerical data for the local exponents $z$. At each point along the path (0) the value of $z$ obtained for $\Gamma$ is smaller than the corresponding value of $z$ obtained for $\ell_{0}$ (see Figs. 16(b) and 17(b)).

For $p=3$ and $p=2$ the variation of the local exponents of the adsorption in the temperature range in between the crossover lines $H_{c r}^{(1)}$ and $H_{c r}^{(2)}$ is less pronounced than that for $p=50$. Near $H_{c r}^{(1)}$ we find saturation for the local exponents $z$ at values which are smaller than the saturation values of $z$ of the corresponding thickness $\ell_{0}$ of the wetting film, i.e., $z \simeq 0.49$ and $z \simeq 0.36$ for $p=3$ and $p=2$, respectively (see Fig. 17(b)). This is consistent with the relation $\Gamma \sim \ell_{0} \Delta m$ and can be explained by the same arguments as used in the previous subsection. We note, that $\Delta m \sim|\tau|^{\beta}$, with $\beta=1 / 8=0.125$ for the $d=2$ Ising model, leads to bigger differences in the local exponents for $\ell_{0}$ and $\Gamma$ than the aforementioned ones observed in the range of their saturation $\left[p=3: z\left(\ell_{0}\right)-\right.$ $z(\Gamma)=0.55-0.49=0.06<0.125 ; p=2: z\left(\ell_{0}\right)-z(\Gamma)=$ $0.44-0.36=0.08<0.125]$. However, the above power laws are satisfied only sufficiently close to $T_{c}$, which is not reached along $H_{c a}(T, L)$ for the finite values of $L$ studied 
here.

\section{SUMMARY AND CONCLUSIONS}

We have studied two-dimensional Ising ferromagnets in strip geometries of width $L$ and with long-ranged boundary fields $\left(V_{j}^{s}=\frac{h_{1}}{j^{p}}, h_{1}>0\right.$ ) (Eqs. (12) and (13)). Based on scaling theory and the density-matrix renormalization-group method we have obtained the following main results:

(1) In Sects. 凹and We have discussed the theoretical framework and the expectations due to scaling theory for the interplay between critical adsorption and complete wetting, including the crossover between them. For semiinfinite systems the corresponding scaling arguments lead to the schematic phase diagram shown in Fig. 1.

(2) The location of the pseudo-phase coexistence line $H_{c a}(T, L ; p)$ of capillary condensation has been determined for various ranges $p$ of the boundary fields (Fig. 31). For positive and parallel surface fields capillary condensation occurs at negative values $H_{c a}(T, L ; p)$ of the bulk field $H$. Increasing the range of the boundary fields shifts the pseudo-phase coexistence line towards more negative values of the bulk field $H$ (see Figs. 3 and 4). Relative to the pseudo-capillary condensation line in the case that the surface fields act only on the two boundary layers $j=1$ and $j=L$, i.e., for $p=\infty, H_{c a}(T, L ; p)$ varies exponentially as function of the range $p$ (Fig. (4).

(3) A critical wetting transition has been identified for $p=2$, i.e., for a boundary field decaying slower than the marginal one $(p=3)$ (see Fig. 5). These findings disprove previous claims by Kroll and Lipowsky [37] that in $d=2$ for $p<3$ there is no critical wetting transition at finite values of $h_{1}$.

(4) Within the accessible range of values for $T$ and $H$ the magnetization profiles both along isotherms (see Figs. 6] and 7) and along the pseudo-capillary condensation line (see Fig. 3) are not slab-like. They exhibit a wide interfacial region and significant tails. Primarily the profiles approach their plateau values in the middle of the strip exponentially as function of the distance from the wall. The width of the emerging interfacial region and the decay length of the tails are proportional to the bulk correlation length and thus grow upon approaching $T_{c}$ or the pseudo-capillary condensation line. In the presence of long-ranged boundary fields the exponential decay of the profiles towards their bulk values $m_{b}(H, T)$ is followed by an algebraic decay $j^{-p}$ which finally is distorted by the presence of the distant wall. Features similar to those of these order parameter profiles have been inferred from neutron reflectrometry for the composition profile of a wetting film in a binary liquid mixture of n-hexane and perfluoro-n-hexane [47].

(5) The variation of the thickness $\ell_{0}$ of the wetting layer along various isotherms (Figs. 8f[10) and along the pseudo-phase coexistence line (Fig. 16) has been analyzed for wide strips and different ranges $p$ of the boundary field. Along both types of path we have found a gradual increase of $\ell_{0}$ upon approaching $T_{c}$. The asymptotic divergence of $\ell_{0}$ along the isotherms is preempted by capillary condensation. As discussed on general grounds at the end of Subsec. VIA3 both in $d=2$ and $d=3$ along the isotherm close to $T_{c}$ the ultimate crossover to the complete wetting behavior (see hatched region and path II in Fig. 1) can only be observed in systems with macroscopically large transverse extensions. Along the isotherms and within the complete wetting regime the suitably defined local exponents of $\ell_{0}$ (Eq. (20) ) tend to approach the values predicted by the corresponding effective interface Hamiltonian, i.e., $\beta_{s}^{c o}=1 / 3$ for $p \geq 3$ and $\beta_{s}^{c o}=1 / p$ for $p<3$ (Figs. 8 and 9). These results further support our findings that a critical wetting transition exists also for the values $p=2$ and 1.5 of the decay exponent of the boundary field, i.e., smaller than the marginal case $p=3$. Along the pseudo-phase coexistence line $H_{c a}(T, L)$ the variation of the thickness of the wetting film is determined by the functional form of the temperature dependence of $H_{c a}(T, L)$ and for $p>2$ it agrees with the predictions of an effective interface Hamiltonian (Eq. (27) and Fig. 16(a)). For long-ranged boundary fields with $p=3$ and $p=2$, the increase of $\ell_{0}$ within a certain range of temperature can be described by a power law with an effective exponent which is, however, not universal. It varies from $c a 0.55$ for $p=3$ to $c a$ 0.44 for $p=2$ (Fig. 16(b)).

(6) For $p=50,4,3$, and 2 the adsorption $\Gamma$ (Eqs. (3) and (23) ) has been calculated along various isotherms (see Figs. 11] 13). Along these thermodynamic paths, over a wide range of the bulk field $H$ the adsorption $\Gamma$ exhibits a continuous increase upon approaching the pseudo-phase coexistence line $H_{c a}(T, L ; p)$. Within the accessible range of values for $H$ this increase cannot be described by simple power laws. Only very close to $H_{c a}(T, L ; p)$ the local exponents of $\Gamma$ start to approach their predicted values, i.e., $\beta_{s}^{c o}(p \geq 3)=1 / 3$ and $\beta_{s}^{c o}(p=2)=1 / 2$ for complete wetting (see Subsec. ЏA and Figs. 11 and 12) $)$ and $(\nu-\beta) / \Delta=7 / 15$ for critical adsorption (see Eq. (2), Subsec. IIB, and Fig. 13). However, these results are not entirely conclusive because the asymptotic regimes are preempted by capillary condensation so that one is unable to detect the crossover from critical adsorption to complete wetting which is expected to occur sufficiently close to bulk coexistence for isotherms with $T$ close to $T_{c}$. For the low temperature isotherms, which lie entirely within the complete wetting regime, the cases $p=1.5,2$, i.e., for $p$ smaller than the marginal value $p=3$, differ distinctly from the cases $p \geq 3$ (see Subsec. II A and Fig. 11), which is a clear indication of the non-universality of complete wetting for these cases. For $p=1.5$ the long-ranged part of the boundary field is relevant in the RG sense and the behavior of $\Gamma$ is non-universal along the critical isotherm (see Fig. 13(b)).

(7) The finite-size scaling predictions for the capillary condensation line (see Eqs. (24) and (25) as well as Figs.2 
and 14) are satisfied for both short-ranged $(p=50)$ and long-ranged $(p=3)$ boundary fields.

(8) For $p=50,3$, and 2 the magnetization profiles (Fig. 15) and the adsorption $\Gamma$ (Fig. 17) have been calculated along the pseudo-capillary condensation line $H_{c a}(T, L)$. Over a wide range of temperatures below $T_{c}$, $\Gamma$ increases gradually (see Fig. 17). Near criticality its increase cannot, however, be described by a simple power law behavior. The reason is that along this thermodynamic path the temperature dependence of $\Gamma$ is determined also by the temperature variation of $H_{c a}(T, L)$, similarly to the thickness $\ell_{0}$ of the wetting film which is approximately related to $\Gamma$ as $\Gamma \simeq \ell_{0} \Delta m$ where $\Delta m(T)$ is the temperature dependent difference between the magnetization of the spin up and the spin down bulk phases. Our theoretical results cannot be directly compared with the experimental findings in Refs. 15] and [16], in which the thickness of the wetting layer [15] and the adsorption [16] were measured along the paths corresponding to the path (1) in Fig. 1, i.e., in these experiments the capillary condensation was absent. The data obtained from both experiments show that the behavior of $\ell_{0}$ and $\Gamma$ can be described by a power law but with an effective exponent which differs from both that for complete wetting and that for critical adsorption. Similarly, in our theoretical analysis of two-dimensional Ising strips, for long-ranged boundary fields with $p=3$ and $p=2$ the increase of $\Gamma$, for still sufficiently large reduced temperatures $|\tau|$, can be described by a power law with an effective exponent, here with a value of $c a$. 0.44 for $p=3$ and of $c a$. 0.36 for $p=2$ (Fig. 17).

(9) Our results indicate that the asymptotic behavior for $H \rightarrow 0$ occurs in a much narrower regime than one would expect and therefore requires much larger system sizes to suppress capillary condensation sufficiently in order to reach the asymptotic regime. One indication for having entered the asymptotic regime could be that a fully developed wetting layer has been formed. For the system sizes $L$ and thus the values of $H$ accessible in our calculations this does not yet fully occur.

\section{Acknowledgments}

A. D. thanks the Wrocław Centre for Networking and Computing (grant No. 82) and the Computing Center of the Institute of Low Temperature and Structure Research PAS for access to their computing facilities.
[1] K. Binder, in Phase Transitions and Critical Phenomena, edited by C. Domb and J. L. Lebowitz (Academic, London, 1983), Vol. 8, p. 1.

[2] H. W. Diehl, in Phase Transitions and Critical Phenomena, edited by C. Domb and J. L. Lebowitz (Academic, London, 1986), Vol. 10, p. 76.

[3] S. Dietrich, in Phase Transitions and Critical Phenomena, edited by C. Domb and J. L. Lebowitz (Academic, London, 1988), Vol. 12, p. 1.

[4] Y. Xia, B. Gates, Y. Yin, and Y. Lu, Adv. Mater. 12, 693 (2000).

[5] E. Bertrand and J. Petrol. Sci. Eng. 33, 217 (2002).

[6] P. G. de Gennes, Rev. Mod. Phys. 57, 827 (1985).

[7] P. Tabeling, Microfluidics (EDP Sciences, Paris, 2004).

[8] Supercritical Fluids - Fundamentals and Applications, edited by E. Kiran, P. G. Debenedetti and C. J. Peters, Proceedings of the NATO Advanced Study Institute, NATO Science Series E, Vol. 366, (Kluwer, Dordrecht, 2000).

[9] R. Riehn and R. H. Austin, Anal. Chem. 78, 5933 (2006).

[10] P. Pfeuty and G. Toulouse, Introduction to the Renormalization Group and to Critical Phenomena (Wiley, London, 1977).

[11] A. Pelissetto and E. Vicari, Phys. Rep. 368, 549 (2002).

[12] P. Tarazona, M. M. Telo da Gama and M. Robert, J. Chem. Phys. 86, 1521 (1987).

[13] S. Dietrich, in Phase Transitions in Surface Films 2, edited by H. Taub, G. Torzo, H. J. Lauter, and S. C. Jr. Fain, Proceedings of the NATO Science Series B (Plenum, New York, 1991), Vol. 267, p. 391, and references therein.

[14] M. M. Telo da Gama and U. Marini Bettolo Marconi,
Physica A 171, 69 (1991).

[15] D. Fenistein, D. Bonn, S. Rafaï, G. H. Wegdam, J. Meunier, A. O. Parry, and M. M. Telo da Gama, Phys. Rev. Lett. 89, 096101 (2002).

[16] J. Bowers, A. Zarbakhsh, A. Querol, H. K. Christenson, I. A. McLure, and R. Cubitt, J. Chem. Phys. 121, 9058 (2004).

[17] J. R. Silvius, Thermotropic phase transitions of pure lipids in model membranes and their modifications by membrane proteins (John Wiley, New York, 1982).

[18] A. R. Honerkamp-Smith, P. Cicuta, M. D. Collins, S. L. Veatch, M. den Nijs, M. Schick, and S. L. Keller, Biophys. J. 95, 236 (2008).

[19] S. R. White, Phys. Rev. Lett. 69, 2863 (1992); S. R. White, Phys. Rev. B 48, 10345 (1993).

[20] T. Nishino, J. Phys. Soc. Jpn. 64, 3598 (1995).

[21] Lecture Notes in Physics, edited by I. Peschel, X. Wang, M. Kaulke, and K. Hallberg (Springer, Berlin, 1999), Vol. 528.

[22] U. Schollwoeck, Rev. Mod. Phys. 77, 259 (2005).

[23] D. Beysens and D. Estève, Phys. Rev. Lett. 54, 2123 (1985); B. M. Law, J.-M. Petit, and D. Beysens, Phys. Rev. E 57, 5782 (1998).

[24] H. Guo, T. Narayanan, M. Sztuchi, P. Schall, and G. H. Wegdam, Phys. Rev. Lett. 100, 188303 (2008).

[25] M. Schick, in Liquids at Interfaces, Proceedings of the Les Houches Summer School in Theoretical Physics, Session XLVIII, edited by J. Charvolin, J. F. Joanny, and J. ZinnJustin (North-Holland, Amsterdam, 1990), p. 415.

[26] R. Lipowsky, Phys. Rev. B 32, 1731 (1985).

[27] M. E. Fisher and P. G. de Gennes, C. R. Acad. Sci. Ser. B 287, 207 (1978). 
[28] G. Flöter and S. Dietrich, Z. Phys. B 97, 213 (1995).

[29] R. Pandit, M. Schick, and M. Wortis, Phys. Rev. B 26, 5112 (1982).

[30] T. Getta and S. Dietrich, Phys. Rev. E 47, 1856 (1993).

[31] V. Privman and M. E. Fisher, J. Stat. Phys. 33, 385 (1983), and references therein.

[32] K. Binder, Annu. Rev. Mater. Res. 38, 123 (2008).

[33] W. Thomson, Phil. Mag. 42, 448 (1871).

[34] R. Evans, J. Phys.: Condens. Matter 2, 8989 (1990), and references therein.

[35] M. E. Fisher and H. Nakanishi, J. Chem. Phys. 75, 5857 (1981); H. Nakanishi and M. E. Fisher, ibid 78, 3279 (1983).

[36] A. Maciołek, A. Drzewiński, and R. Evans, Phys. Rev. E 64, 056137 (2001).

[37] D. M. Kroll and R. Lipowsky, Phys. Rev. B 28, 5273 (1983).

[38] V. Privman and N. M Ŝvrakiĉ, Phys. Rev. B 37, 5974 (1988).
[39] A. Drzewiński and K. Szota, Phys. Rev. E 71, 056110 (2005).

[40] E. V. Albano, K. Binder, and W. Paul, J. Phys.: Condens. Matter 12, 2701 (2000).

[41] A. De Virgiliis, E. V. Albano, M. Müller, and K. Binder, J. Phys.: Condens. Matter 17, 4579 (2005).

[42] A. O. Parry and R. Evans, Phys. Rev. Lett. 64, 439 (1990).

[43] D. B. Abraham, Phys. Rev. Lett. 44, 1165 (1980).

[44] M. Napiórkowski, W. Koch, and S. Dietrich, Phys. Rev. A 45, 5760 (1992).

[45] A. O. Parry and R. Evans, Phys. Rev. A 46, 5282 (1992).

[46] K. Binder, D. Landau, and M. Müller, J. Stat. Phys. 110, 1411 (2003).

[47] J. Bowers, A. Zarbakhsh, I. A. McLure, J. R. P. Webster, R. Steitz, and H. K. Christenson, J. Phys. Chem. C 111, 5569 (2007). 